\title{
A Merger\#driven Scenario for Cosmological Disk Galaxy Formation
}

\section{Citation}

Robertson, Brant, James S. Bullock, Thomas J. Cox, Tiziana Di Matteo, Lars Hernquist, Volker Springel, and Naoki Yoshida. 2006. "A Merger\#driven Scenario for Cosmological Disk Galaxy Formation." The Astrophysical Journal 645 (2): 986-1000. https://doi.org/10.1086/504412.

\section{Permanent link}

http://nrs.harvard.edu/urn-3:HUL.InstRepos:41381704

\section{Terms of Use}

This article was downloaded from Harvard University's DASH repository, and is made available under the terms and conditions applicable to Other Posted Material, as set forth at http:// nrs.harvard.edu/urn-3:HUL.InstRepos:dash.current.terms-of-use\#LAA

\section{Share Your Story}

The Harvard community has made this article openly available.

Please share how this access benefits you. Submit a story. 
Draft Version September 9, 2018

Preprint typeset using $\mathrm{LAT}_{\mathrm{E} X}$ style emulateapj v. 9/08/03

\title{
A MERGER-DRIVEN SCENARIO FOR COSMOLOGICAL DISK GALAXY FORMATION
}

\author{
Brant Robertson ${ }^{1,6}$, James S. Bullock ${ }^{2}$, Thomas J. Cox ${ }^{1}$, \\ Tiziana Di Matteo ${ }^{3}$, Lars Hernquist ${ }^{1}$, Volker Springel ${ }^{4}$, NaOKi Yoshida $^{5}$ \\ Draft version September 9, 2018
}

\begin{abstract}
The violent hierarchical nature of the $\Lambda$-Cold Dark Matter cosmology poses serious difficulties for the formation of disk galaxies. To help resolve these issues, we describe a new, merger-driven scenario for the cosmological formation of disk galaxies at high redshifts that supplements the standard model based on dissipational collapse. In this picture, large gaseous disks may be produced from highangular momentum mergers of systems that are gas-dominated, i.e. $\mathcal{M}_{\text {gas }} /\left(\mathcal{M}_{\text {gas }}+\mathcal{M}_{\star}\right) \gtrsim 0.5$ at the height of the merger. Pressurization from the multiphase structure of the interstellar medium prevents the complete conversion of gas into stars during the merger, and if enough gas remains to form a disk, the remnant eventually resembles a disk galaxy. We perform numerical simulations of galaxy mergers to study how supernovae feedback strength, supermassive black hole growth and feedback, progenitor gas fraction, merger mass-ratio, and orbital geometry impact the formation of remnant disks. We find that disks can build angular momentum through mergers and the degree of rotational support of the baryons in the merger remnant is primarily related to feedback processes associated with star formation. Nearly every simulated gas-rich merger remnant contains rapidlyrotating stellar substructure, while disk-dominated remnants are restricted to form in mergers that are gas-dominated at the time of final coalescence. Typically, gas-dominated mergers require extreme progenitor gas fractions $\left(f_{\text {gas }}>0.8\right)$. We also show that the formation of rotationally-supported stellar systems in mergers is not restricted to idealized orbits, and both gas-rich major and minor mergers can produce disk-dominated stellar remnants. We suggest that the hierarchical nature of the $\Lambda$-Cold Dark Matter cosmology and the physics of the interstellar gas may act together to form spiral galaxies by building the angular momentum of disks through early, gas-dominated mergers. Our proposed scenario may be especially important for galaxy formation at high redshifts, where gas-dominated mergers are believed to be more common than in the local Universe.
\end{abstract}

Subject headings: galaxies: formation - galaxies: evolution

\section{INTRODUCTION}

The conventional theory for the origin of disk galaxies in Cold Dark Matter (CDM) cosmologies involves the dissipational collapse of gas inside relaxed dark matter halos formed through hierarchical clustering (White \& Rees 1978; Blumenthal et al. 1984). This theory endows forming disk galaxies with kinematic properties inherited from the halos that host them (Fall \& Efstathiou 1980). Early simulations of cosmological structure formation (Barnes \& Efstathiou 1987) verified that during linear growth, the angular momentum of halos grows according to perturbation theory (Peebles 1969), and by incorporating these results subsequent work explained the flat rotation curves and sizes of disk galaxies through the combination of gas dissipation, halo spin, and the adiabatic response of dark matter to baryonic infall (Blumenthal et al. 1986; Mo et al.1998). The initial suc-

\footnotetext{
${ }^{1}$ Harvard-Smithsonian Center for Astrophysics, 60 Garden St. Cambridge, MA 02138, USA

${ }^{2}$ Department of Physics \& Astronomy, University of California, Irvine, CA 92697, USA

3 Carnegie-Mellon University, Department of Physics, 5000 Forbes Ave., Pittsburgh, PA 15213, USA

4 Max-Planck-Institut für Astrophysik, Karl-SchwarzschildStraße 1, 85740 Garching bei München,

Germany

5 Department of Physics, Nagoya University, Nagoya 464-8602, Japan

6 brobertson@cfa.harvard.edu
}

cess of the dissipational collapse model led to its establishment as the favored theory for the formation of disk galaxies. However, numerical modeling revealed that the theory was incomplete.

While smooth, dissipational collapse may be relevant to disk galaxy formation in quiet environments, simulations and semi-analytic modeling have demonstrated that it is rare for halos to acquire most of their angular momentum via the quiescent accretion of tidally torqued material (Vitvitska et al. 2002; Maller \& Dekel 2002; D'Onghia \& Burkert 2004). Simulation and observational work has shown that the specific angular momentum content of dark matter halos differs from exponential disks (Bullock et al. 2001; van den Bosch et al. 2001, 2002) as halos contain comparatively more low angular momentum material that might produce bulges or overly centrally concentrated disks (van den Bosch 1998, 2001). Furthermore, simulations of mass accretion onto galaxies indicate that the hierarchical nature of the $\Lambda \mathrm{CDM}$ cosmology leads to the destruction of disks when dissipative effects are neglected (Toth \& Ostriker 1992; Quinn et al. 1993; Walker et al. 1996: Velazquez \& White 1999). Models of galaxy collisions including dissipation (Hernquist 1989; Barnes \& Hernquist 1991, 1996) show that gas can loose angular momentum owing to gravitational torques during these events. In cases when the interstellar medium (ISM) is isothermal and relatively cold and the gas frac- 
tion of the galaxies is small ( $\sim 10 \%$ of the baryons), the subsequent inflow of gas into the centers of the merger remnants forms a roughly spherical stellar distribution through a luminous starburst (Mihos \& Hernquist 1994d, 1996), leaving objects that have essentially no extended stellar disks. Cosmological simulations of disk formation with similar physics produce galaxies that are too centrally concentrated, form too many stars, contain overly dominant bulges, and lack angular momentum compared with nearby spirals (Katz \& Gunn 1991; Katz et al. 1992; Navarro \& White 1994; Steinmetz \& Muller 1995; Navarro \& Steinmetz 2000).

Efforts to resolve these discrepancies in a cosmological context have mainly relied on increasing the impact of feedback from star formation (Weil et al. 1998; Sommer-Larsen et al. 1999, 2003; Thacker \& Couchman 2000, 2001; Abadi et al. 2003a; Governato et al. 2004; Robertson et al. 2004; Okamoto et al.|2005). Cosmological simulations that utilize strong feedback from star formation in the form of a pressurized ISM to produce disk galaxies either produce too few exponential disks compared with observations (Robertson et al. 2004) or yield galaxies that still contain a significant bulge component (Abadi et al. 2003a). While these recent simulations have had some success, taken together the results suggest that the theory of structure formation remains faced with an apparent incompatibility between models of disk formation and the hierarchical growth of structure.

To help resolve this incompatibility, we propose a new, merger-driven theory for the formation of disk galaxies at high redshifts that supplements standard dissipational collapse. In our scenario, gas-dominated mergers, where the interacting systems have a gas fraction $f_{\text {gas }} \equiv \mathcal{M}_{\text {gas }} /\left(\mathcal{M}_{\text {gas }}+\mathcal{M}_{\star}\right) \gtrsim 0.5$ at the height of the merger, allow for the formation of rotationallysupported disks in remnants if energetic feedback mechanisms limit the conversion of gas into stars. Gas pressurization from a multiphase ISM by feedback from star formation (McKee \& Ostriker 1977; Springel \& Hernquist 2003) stabilizes gas disks and allows mergers between gas-dominated galaxies to produce large, smoothly distributed, rapidly rotating stellar disks. Our proposed scenario builds upon the results of previous simulations of remnant disk formation in mergers by Barnes (2002) and Springel \& Hernquist (2005).

The merger-driven scenario for disk formation transitions naturally into the merger-driven scenario for elliptical galaxy formation (e.g. Toomre 1977) as star formation in galaxies over time reduces the gas content of progenitors and prevents the further occurrence of mergers involving gas-dominated systems. Mergers between gas-rich disk galaxies at intermediate redshifts $(z \approx 1-3)$ are still expected to produce realistic elliptical galaxies nearly universally (see e.g. Robertson et al. 2006), as prior quiescent star formation and interactions will decrease their gas content below the necessary $f_{\text {gas }} \gtrsim 0.5$ needed to produce a disk-dominated remnant.

Below we describe the simulation methodology used to demonstrate the merger-driven formation of disk galaxies (\$2), including our methods for studying the effects of feedback (2.1), orbital geometry (2.2), gas fraction (\$2.3), merger mass-ratio (\$2.4), and progenitor mass (\$2.5) on remnant properties. Our method for analyzing the structural and kinematic properties of the remnants is detailed in $\$ 2.6$ and the results of the numerical experiments are presented in \$3. We discuss and summarize our results in 4 and $\$ 5$

\section{METHODOLOGY}

To demonstrate the feasibility of our scenario for merger-driven disk formation, we have performed a suite of merger simulations of isolated, gas-rich disk galaxies, with and without feedback from accretion onto supermassive black holes (BHs). The simulations use Smoothed Particle Hydrodynamics (SPH) (Lucv 1977; Gingold \& Monaghan 1977) to evolve the gas and incorporate a multiphase ISM model (Yepes et al. 1997; Hultman \& Pharasvn 1999; Springel \& Hernquist 2003; Springel et al. 2005b) where we control the pressurization $q_{\mathrm{EOS}}$ of star-forming gas by interpolating between isothermal gas $\left(q_{\mathrm{EOS}}=0\right)$ with a temperature $T_{\text {eff }}=10^{4} \mathrm{~K}$ and a stiffer multiphase medium $\left(q_{\mathrm{EOS}}=\right.$ 1 ) with a higher effective temperature $T_{\text {eff }} \approx 10^{5} \mathrm{~K}$ by varying the equation of state (EOS; for details see Springel et al. 2005b). Star formation in the dense ISM $\left(\Sigma>10 M_{\odot} \mathrm{pc}^{-2}\right)$ occurs on a timescale chosen to match observations (Kennicutt 1998). BH growth is modeled (Springel et al. 2005b) by spherical accretion onto $\mathrm{BH}$ "sink" particles, with a fraction $\epsilon_{\mathrm{f}}$ of the released gravitational potential energy of the accreted gas being deposited as thermal feedback into the gas surrounding the BH. For the simulations and accretion model considered here, $\epsilon_{\mathrm{f}}=\eta_{\text {therm }} \epsilon_{\mathrm{r}}=0.005$, where $\epsilon_{\mathrm{r}} \approx 0.1$ is the fraction of rest mass energy radiated during the growth of the $\mathrm{BH}$ (fixed by standard accretion theory) and the thermal coupling efficiency $\eta_{\text {therm }}=0.05$ is chosen to reproduce the local $M_{\mathrm{BH}}-\sigma$ relation (Di Matteo et al. 2005).

We consider two merger progenitors, a $V_{\text {vir }}=160 \mathrm{~km}$ $\mathrm{s}^{-1}$ system (Springel \& Hernauist 2005) with a rotation curve and virial mass $M_{\text {vir }}=9.5 \times 10^{11} h^{-1} M_{\odot}$ similar to the Milky Way and a smaller, $V_{\text {vir }}=80 \mathrm{~km} \mathrm{~s}^{-1}$ system with virial mass $M_{\text {vir }}=1.2 \times 10^{11} h^{-1} M_{\odot}$. The galaxies are comprised of exponential gas and stellar disks embedded in dark matter halos with a Hernquist (1990) profile having scale lengths corresponding to a Navarro-FrenkWhite (Navarro et al. 1997) concentration of $c_{\mathrm{NFW}}=9$. The progenitors each contain $N_{\text {gas }}=40000$ gas, $N_{\text {stars }}=$ 40000 stellar, and $N_{\mathrm{dm}}=180000$ dark matter halo particles, and may also include a BH seed of $10^{5} h^{-1} M_{\odot}$ that is allowed to accrete in the manner described above. Once the $\mathrm{BH}$ particles fall within our force resolution limit, the sink particles combine into a single supermassive $\mathrm{BH}$.

To characterize the conditions under which our proposed scenario for merger-driven disk formation is most successful, we simulate a variety of merger scenarios varying the ISM pressurization $\left(q_{\mathrm{EOS}}\right)$, gas fraction $\left(f_{\text {gas }}\right)$, merger mass-ratio, disk orientations, and orbits. The complete suite of simulations is described below, and a summary is provided in Table 1] For convenience we will refer to each model with a two letter designation, with the first letter signifying the gas fraction $f_{\text {gas }}$ and the second letter noting the ISM pressurization $q_{\text {EOS }}$. When necessary, we add suffixes to describe e.g. orbital or merger mass-ratio variations. The simulation designations are detailed in the following sections.

\subsection{Feedback Study}


TABle 1. Merger Models

\begin{tabular}{|c|c|c|c|c|c|c|c|c|c|c|c|}
\hline Model & $\begin{array}{c}V_{\mathrm{vir}, \mathrm{A}} \\
{\left[\mathrm{km} \mathrm{s}^{-1}\right]}\end{array}$ & $\begin{array}{c}V_{\mathrm{vir}, \mathrm{B}} \\
{\left[\mathrm{km} \mathrm{s}^{-1}\right]}\end{array}$ & $\overline{f_{\text {gas }}}$ & $q_{\mathrm{EOS}}$ & $\begin{array}{c}r_{\text {peri }} \\
{\left[h^{-1} \mathrm{kpc}\right]}\end{array}$ & $\begin{array}{c}r_{\text {init }} \\
{\left[h^{-1} \mathrm{kpc}\right]}\end{array}$ & $\begin{array}{c}\theta_{1} \\
\text { [degrees] }\end{array}$ & $\begin{array}{c}\phi_{1} \\
{[\text { degrees] }}\end{array}$ & $\begin{array}{c}\theta_{2} \\
\text { [degrees] }\end{array}$ & $\begin{array}{c}\phi_{2} \\
\text { [degrees] }\end{array}$ & $\overline{\mathrm{BH}}$ \\
\hline GA & 160 & 160 & 0.99 & 0.1 & 6.0 & 70.0 & 0 & 0 & 0 & 0 & $Y$ \\
\hline GB & 160 & 160 & 0.99 & 0.25 & 6.0 & 70.0 & 0 & 0 & 0 & 0 & $\mathrm{Y}$ \\
\hline $\mathrm{GC}$ & 160 & 160 & 0.99 & 0.5 & 6.0 & 70.0 & 0 & 0 & 0 & 0 & $\mathrm{Y}$ \\
\hline GD & 160 & 160 & 0.99 & 0.75 & 6.0 & 70.0 & 0 & 0 & 0 & 0 & $\mathrm{Y}$ \\
\hline GE & 160 & 160 & 0.99 & 1.0 & 6.0 & 70.0 & 0 & 0 & 0 & 0 & $\mathrm{Y}$ \\
\hline \multicolumn{12}{|c|}{ No BHs } \\
\hline GAn & 160 & 160 & 0.99 & 0.1 & 6.0 & 70.0 & 0 & 0 & 0 & 0 & $\mathrm{~N}$ \\
\hline GBn & 160 & 160 & 0.99 & 0.25 & 6.0 & 70.0 & 0 & 0 & 0 & 0 & $\mathrm{~N}$ \\
\hline $\mathrm{GCn}$ & 160 & 160 & 0.99 & 0.5 & 6.0 & 70.0 & 0 & 0 & 0 & 0 & $\mathrm{~N}$ \\
\hline GDn & 160 & 160 & 0.99 & 0.75 & 6.0 & 70.0 & 0 & 0 & 0 & 0 & $\mathrm{~N}$ \\
\hline GEn & 160 & 160 & 0.99 & 1.0 & 6.0 & 70.0 & 0 & 0 & 0 & 0 & $\mathrm{~N}$ \\
\hline \multicolumn{12}{|c|}{$r_{\text {peri }}$ Variations } \\
\hline GCrA & 160 & 160 & 0.99 & 0.5 & 2.7 & 70.0 & 0 & 0 & 0 & 0 & $\mathrm{Y}$ \\
\hline $\mathrm{GCrB}$ & 160 & 160 & 0.99 & 0.5 & 16.0 & 70.0 & 0 & 0 & 0 & 0 & $\mathrm{Y}$ \\
\hline $\mathrm{GCrC}$ & 160 & 160 & 0.99 & 0.5 & 32.0 & 70.0 & 0 & 0 & 0 & 0 & $\mathrm{Y}$ \\
\hline GCrD & 160 & 160 & 0.99 & 0.5 & 48.0 & 140.0 & 0 & 0 & 0 & 0 & $\mathrm{Y}$ \\
\hline \multicolumn{12}{|c|}{ Orbital Variations } \\
\hline $\mathrm{GCoC}$ & 160 & 160 & 0.99 & 0.5 & 6.0 & 70.0 & 180 & 0 & 180 & 0 & $\mathrm{Y}$ \\
\hline GCoE & 160 & 160 & 0.99 & 0.5 & 6.0 & 70.0 & 30 & 60 & -30 & 45 & $\mathrm{Y}$ \\
\hline $\mathrm{GCoF}$ & 160 & 160 & 0.99 & 0.5 & 6.0 & 70.0 & 60 & 60 & 150 & 0 & $\mathrm{Y}$ \\
\hline $\mathrm{GCoO}$ & 160 & 160 & 0.99 & 0.5 & 6.0 & 70.0 & -109 & 30 & 71 & -30 & $\mathrm{Y}$ \\
\hline GCoP & 160 & 160 & 0.99 & 0.5 & 6.0 & 70.0 & -109 & 90 & 180 & 0 & $\mathrm{Y}$ \\
\hline \multicolumn{12}{|c|}{$f_{\text {gas }}$ Variations } \\
\hline$\overline{D C}$ & 160 & 160 & 0.4 & 0.5 & 6.0 & 70.0 & 0 & 0 & 0 & 0 & $\mathrm{Y}$ \\
\hline EC & 160 & 160 & 0.6 & 0.5 & 6.0 & 70.0 & 0 & 0 & 0 & 0 & $\mathrm{Y}$ \\
\hline FC & 160 & 160 & 0.8 & 0.5 & 6.0 & 70.0 & 0 & 0 & 0 & 0 & $\mathrm{Y}$ \\
\hline \multicolumn{12}{|c|}{ Minor Mergers } \\
\hline $\mathrm{DCm}$ & 160 & 80 & 0.4 & 0.5 & 6.0 & 70.0 & 0 & 0 & 0 & 0 & $\mathrm{Y}$ \\
\hline $\mathrm{ECm}$ & 160 & 80 & 0.6 & 0.5 & 6.0 & 70.0 & 0 & 0 & 0 & 0 & $\mathrm{Y}$ \\
\hline FCm & 160 & 80 & 0.8 & 0.5 & 6.0 & 70.0 & 0 & 0 & 0 & 0 & $\mathrm{Y}$ \\
\hline \multicolumn{12}{|c|}{$V_{\text {vir }}=80 \mathrm{~km} \mathrm{~s}^{-1}$ Progenitors } \\
\hline DCs & 80 & 80 & 0.4 & 0.5 & 6.0 & 70.0 & 0 & 0 & 0 & 0 & $\bar{Y}$ \\
\hline ECs & 80 & 80 & 0.6 & 0.5 & 6.0 & 70.0 & 0 & 0 & 0 & 0 & $Y$ \\
\hline FCs & 80 & 80 & 0.8 & 0.5 & 6.0 & 70.0 & 0 & 0 & 0 & 0 & $\mathrm{Y}$ \\
\hline
\end{tabular}

To study the impact of energetic feedback mechanisms on the formation of disks in gas-rich mergers, we perform 12 simulations of major mergers between $V_{\text {vir }}=160$ $\mathrm{km} \mathrm{s}^{-1}$ galaxies with disks consisting almost entirely of gas $\left(f_{\text {gas }}=0.99\right.$, "G" runs). The ISM pressurization is incrementally increased over the values $q_{\mathrm{EOS}}=0.1$, $0.25,0.5,0.75$, and 1.0 , stiffening the equation of state of the ISM and increasing the sound speed of the gas at a given density and temperature. The simulations are referred to as models GA-GE in Table 1 We perform each simulation twice, once with and once without the inclusion of growing supermassive BHs in the progenitor systems. The models without $\mathrm{BHs}$ are listed with the suffix " $\mathrm{n}$ " in Table 1] and the model GCn $\left(f_{\text {gas }}=0.99\right.$, $\left.q_{\mathrm{EOS}}=0.5\right)$ corresponds to the simulation performed by Springel \& Hernquist (2005).

The galaxies merge on coplanar, prograde orbits with an initial separation $r_{\text {init }}=70 h^{-1} \mathrm{kpc}$ and pericentric distance $r_{\text {peri }}=6 h^{-1} \mathrm{kpc}$. The orbit for the feedback study runs was chosen to reproduce the encounter simulated by Springel \& Hernquist (2005). By using the same orbit for these simulations, we explore the importance of feedback for the formation of disks in mergers separate from the role of angular momentum. We explore the role of angular momentum in producing remnant disks in mergers through additional simulations described in $\$ 2.2$

\subsection{Orbital Study}

The original orbital and disk angular momentum of the progenitors will affect the ability of disks to form from mergers as angular momentum present in residual gas left over from the merger can lead to rapidly rotating structures in the remnant. The formation of remnant stellar disks have been demonstrated in interactions with prograde, coplanar orbits (Springel \& Hernquist 2005), and in simulations without star formation gaseous remnant disks have been shown to form in mergers with both polar and inclined orbits (Barnes 2002).

To judge the importance of orbital and disk angular momentum on a merger-driven scenario for disk galaxy formation, we perform a study of 9 additional simula- 
tions. In four models, we vary the pericentric passage distance $r_{\text {peri }}$ of the merger to alter the orbital angular momentum of the interaction. Typically, increasing $r_{\text {peri }}$ will increase the angular momentum of the orbit and lead to larger, more rotationally-supported disks. However, mergers with larger $r_{\text {peri }}$ undergo inefficient dynamical friction, avoid strong angular momentum loss during the first passage, and therefore take longer to merge. Quiescent star formation will reduce the effective gas fraction of the progenitors before the disks merge in these cases, and may lead to larger stellar spheroids. These two competing effects will be present in cosmological environs to some degree, and for this reason we do not attempt to adjust for the decrease in gas fraction in wide-orbit mergers. In addition to a coplanar orbit with $r_{\text {peri }}=6 h^{-1}$ $\mathrm{kpc}$, which is approximately twice the progenitor disk scale length $R_{\mathrm{d}}$, we simulate one model with a smaller pericentric passage distance $\left(r_{\text {peri }}=R_{\mathrm{d}}\right.$, model GCrA in Table (1) and three with larger pericentric passage distances $\left(r_{\text {peri }}=0.1 R_{\text {vir }}, 0.2 R_{\text {vir }}\right.$, and $0.4 R_{\text {vir }}$, models GCrB-GCrD in Table 10.

In the remaining five models of the orbital study, we vary the orientation of the progenitor disks. While varying the pericentric passage distance increases the orbital angular momentum, much of this angular momentum must be lost to the dark matter halo through dynamical friction before the disks can merge. The original disk angular momenta of the progenitors may then substantially add to the angular momentum of the remnant disk. In addition to the coplanar orbit considered by Springel \& Hernquist (2005), we select five additional disk orientations from the Barnes (1992) study of equalmass mergers of stellar disk galaxies. These orientations range from retrograde-retrograde coplanar $\left(\theta_{1}=180\right.$, $\phi_{1}=0, \theta_{2}=180, \phi_{2}=0$, model GCoC) to prograderetrograde polar $\left(\theta_{1}=60, \phi_{1}=60, \theta_{2}=150, \phi_{2}=0\right.$, model GCoF), with a complete list provided in Table 1 The results of these simulations are presented in \$3.2

\subsection{Gas Fraction Study}

The production of remnant disks in mergers will depend on the gas fraction $f_{\text {gas }}$ of the progenitor disk systems. Stellar disks present before the merger will be substantially heated during the merger and form stellar spheroids. Only galaxies with significant gas fractions before the merger will be able to re-form gaseous disks after the galaxies collide. To characterize the progenitor gas fraction necessary to form a substantial remnant disk, we perform three simulations with growing BHs, ISM pressurization $q_{\mathrm{EOS}}=0.5$ and gas fractions $f_{\mathrm{gas}}=0.4$, 0.6 , and 0.8 (models DC, EC, and FC in Table 1). The results of these simulations are presented in \$3.3

\subsection{Minor Merger Study}

In CDM cosmologies, the frequency of galaxy mergers typically increases with the mass ratio of the merging pair (see e.g. Lacev \& Cole 1993). As mentioned above, previous theoretical studies have suggested that dissipationless interactions between satellites and disk galaxies can destroy disks even if the satellite mass is relatively small ( a few percent of the disk mass, e.g. Quinn et al. 1993). Minor mergers with progenitor mass ratios of 3 : 1 have been shown to produce small gaseous disks in simulations without star formation (Barnes 2002). In simulations including gas dissipation and star formation, minor mergers have been shown to induce starbursts (e.g Mihos \& Hernquist 1994b). Cosmological simulations suggest that some minor mergers may also add mass to the thick component of disk galaxy systems (Abadi et al. 2003b). Minor mergers may then greatly influence the formation and survival of disk galaxies even though the mass accretion rate from minor mergers is typically smaller than that from major mergers. To address the relevance of minor mergers for remnant disks, we rerun the gas fraction study simulations from 42.3 substituting a $V_{\text {vir }}=80 \mathrm{~km} \mathrm{~s}^{-1}$ galaxy for the second progenitor. These mergers with mass ratios of $m_{2} / m_{1} \sim 8: 1$ will help characterize the influence of minor mergers on remnant disk properties and provide examples of models that approximate cosmologically frequent merger events. The results of these simulations are presented in 3.4

\subsection{Small Progenitor Study}

The scale dependent physics of gas dissipation and star formation may influence the ability of remnant disks to form in mergers. Gas cooling is typically more efficient in smaller mass halos as the virial temperature of those systems approach the regime where hydrogen and helium recombination and collisional ionization enables large cooling rates (see e.g. Black 1981). Gas densities in the disks of low mass systems are typically lower than in more massive systems, leading to longer gas consumption timescales. Quiescent star formation in low mass systems during the phases of the merger when the separation between disks is large and gravitational torques are weak will be lower than for high mass systems, which may correspond to a larger gas fraction in the progenitor disks when the systems actually merge (Robertson et al. 2006).

To gauge the net impact of these effects on the formation of remnant disks in mergers of different mass scales, we perform three additional simulations of equal mass mergers between $V_{\text {vir }}=80 \mathrm{~km} \mathrm{~s}^{-1}$ galaxies wit gas fractions of $f_{\text {gas }}=0.4,0.6$, and 0.8 for the progenitor systems (models DCs-FCs in Table 1). The results of these simulations are presented in 3.5

\subsection{Analysis}

Each simulation is evolved over the timescale needed for the progenitor systems to merge, corresponding to $T=3.0-10.3$ Gyr depending on the orbit. To illustrate the structure of the merging system during the collision, Figure 1 shows the time evolution of the gas in mergers with a strongly pressurized $\left(q_{\mathrm{EOS}}=1.0\right)$ ISM with (model GE, bottom panel) and without (model GEn, top panel) feedback from accretion onto supermassive BHs. Each panel shows a $140 \mathrm{kpc} \times 140 \mathrm{kpc}$ square area around the center of mass of the interacting system. At early times in the merger $(T=0.35 \mathrm{Gyr}$, far-left panels), tidal interactions between the galaxies distort them, but as significant accretion onto the BHs has yet to occur, the overall gas distribution remains similar between the simulations. As the gas in the simulation with BHs (bottom panels) begins to experience thermal feedback from $\mathrm{BH}$ accretion, a hot halo of diffuse, low-angular momentum gas expelled through a wind surrounds the interacting galaxies. By the end of the simulations at $T=2.0 \mathrm{Gyr}$ 


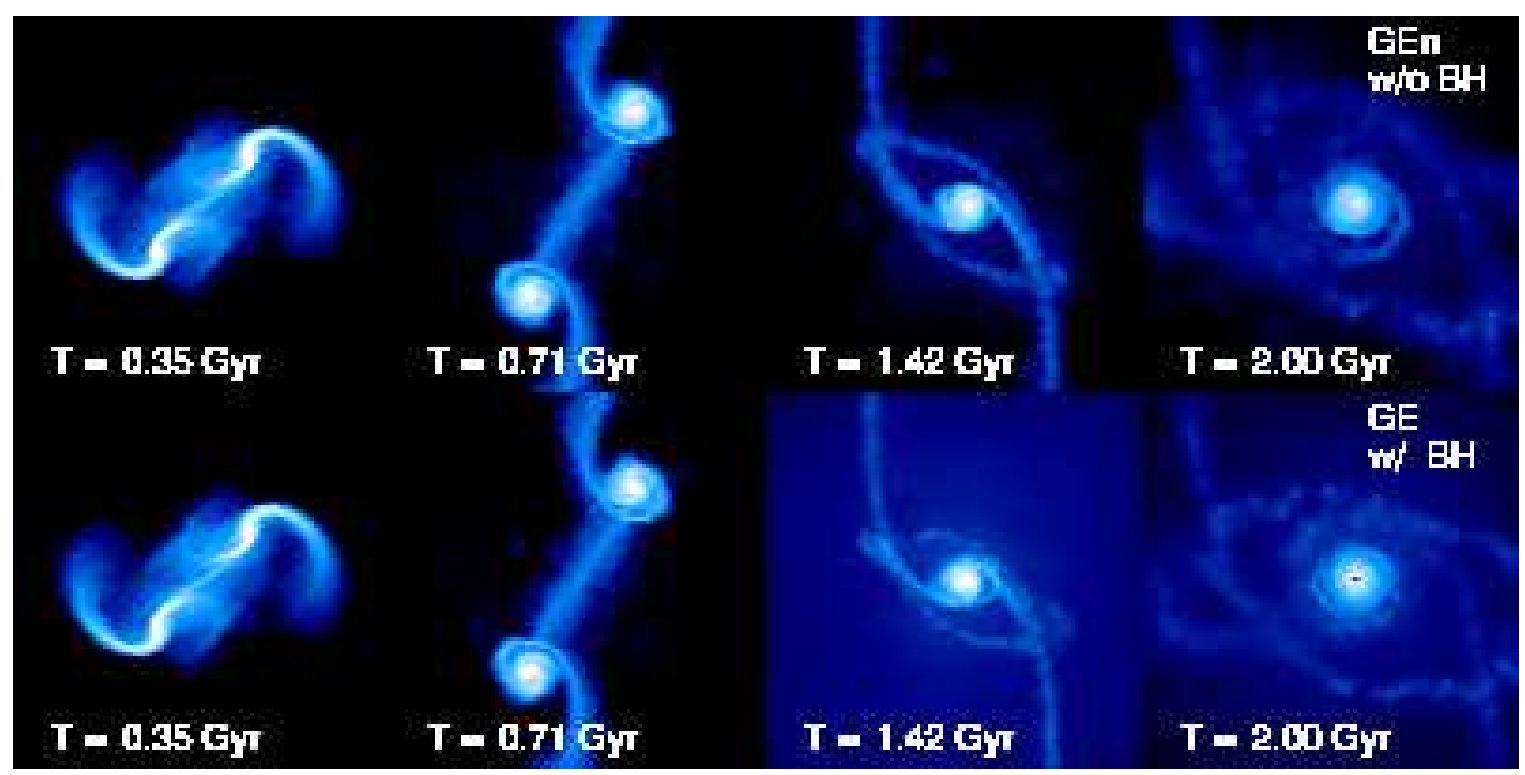

FIG. 1. - Merger of two gas-rich disk galaxies, with (bottom, model GE) and without (top, model GEn) supermassive black hole accretion and feedback. Shown is the gas surface density of the galaxies in each $140 \times 140 \mathrm{kpc}$ square panel, demonstrating that the high-angular momentum merger results in a remnant disk. The effects of black hole feedback can be seen over the 2 Gyr timescale of the merger as a diffuse, hot halo and the lowered central gas density in the remnant (bottom panel).

(far-right panels), rotating gas disks remain, eventually forming stellar disks. The remnant containing a BH has cleared out some gas from the inner regions of the disk, reducing the central stellar density and producing a diffuse hot gas envelope.

After the merger has completed, the structural and kinematic properties of the remnant are measured from the simulation data. The structural components of the merger remnants, which might include a bulge, a thin or thick disk, or an extended spheroid, are comprised of stars that form at characteristically different times during the interaction. The dynamically hot components of the remnant typically form before the final coalescence of the merger, while the stars in the colder components of the remnant mostly form after the height of the merger when the role of tidal forces has decreased. The star formation rate (SFR) of the merging system (see Figure 2. upper left panel) can be used define selection criteria for stars likely to populate separate dynamical components of the remnants based on their formation age. The peak in the SFR that occurs during the height of the merger can be approximated by an exponential rise and decay. In what follows, we identify "old" stars in the remnants as stellar particles forming earlier than before three $e$-folding times before the center of the SFR peak and "new" stars as those forming later than three $e$-folding times after the peak.

The old and new stars in the remnant are then used to measure the separate kinematics of the dynamically hot and cold stellar structures of the remnant. Typically, the old stars in the remnant have relatively low rotational velocities (Figure 2, lower left panel) and are not rotationally supported with the ratio of rotational velocity to velocity dispersion $V_{\text {rot }} / \sigma<1$ (Figure 2 lower right panel). New stars formed in the remnant after the height of the merger typically have high rotational velocities and are rotationally supported. Stars formed during the burst can have kinematic properties intermediate between the old and new stars, and therefore are not analyzed as a separate stellar component.

The mass of the new stellar component forming in the remnant disk can be comparable or large than the old, dynamically heated bulge or extended spheroid. The mass of the stellar structures in the remnant can be measured from the surface mass density profile. After the merger the stellar angular momentum of the remnant is measured and used to define a possible "disk"plane perpendicular to the rotation axis of the remnant. The plane defined normal to the stellar angular momentum vector captures the mean stellar rotation in the remnants we study, and only underestimates rotation when rotationally-supported stellar structures are misaligned with the total stellar angular momentum. The stellar surface mass density of each remnant is then measured in logarithmically-spaced annuli and is modeled by a multi-component system (Figure 2 upper right panel) as a function of radius $r$ that may include an exponential bulge

$$
\Sigma_{\mathrm{b}}(r)=\Sigma_{\mathrm{b}} e^{-r / a_{\mathrm{b}}}
$$

an exponential thin disk

$$
\Sigma_{\mathrm{d}}(r)=\Sigma_{\mathrm{d}} e^{-r / R_{\mathrm{d}}}
$$

an exponential thick disk

$$
\Sigma_{\mathrm{d}, \text { thick }}(r)=\Sigma_{\mathrm{d}, \text { thick }} e^{-r / R_{\mathrm{d}, \text { thick }}}
$$

and an extended stellar spheroid with a de Vaucouleurs (1948) profile

$$
\Sigma_{\mathrm{s}}(r)=\Sigma_{\mathrm{s}} 10^{-3.331\left[\left(r / a_{\mathrm{s}}\right)^{1 / 4}-1\right]}
$$

Each remnant is fitted with individual thin disk, bulgethin disk, bulge-thin disk-thick disk, bulge-thin diskspheroid, and bulge-thin disk-thick disk-spheroid models with the requirements that the bulge scale length 

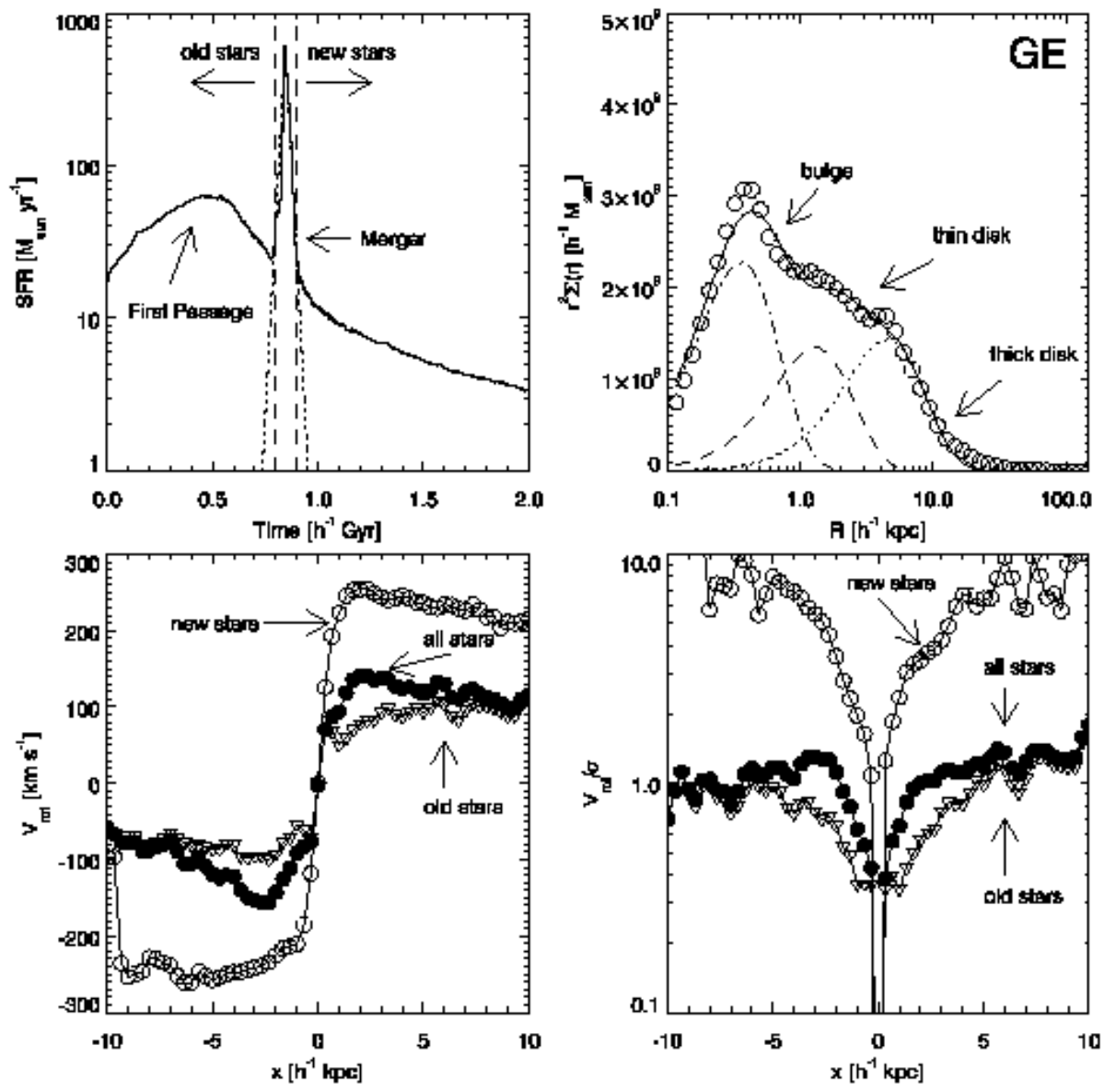

Fig. 2.- Example analysis performed for each merger simulation, shown for an equal mass merger between $V_{\mathrm{vir}}=160 \mathrm{~km} \mathrm{~s}{ }^{-1}$ galaxies with gas-rich disks $\left(f_{\text {gas }}=0.99\right)$, strongly pressurized ISM $\left(q_{\mathrm{EOS}}=1.0\right)$, and growing supermassive black holes (model GE from Table 1 . The peak of the star formation rate (SFR) measured during the simulation is used to identify the height of the merger (upper left panel). An exponential rise and decay is fitted to the SFR peak (dotted line) and used to categorize the stellar content of each remnant based on its formation time. Stars forming before three $e$-folding times prior to the maximum of the SFR rate are labeled as "old" stars (left dashed line), while stars forming three $e$-folding times after the SFR peak are labeled as "new" stars (right dashed line). Typically the new stars forming from remnant disks have large rotational velocities (lower left panel) and are rotationally supported with the ratio of rotationally velocity to velocity dispersion $V_{\text {rot }} / \sigma>1$ (lower right panel). The surface mass density $\Sigma(r)$ (upper right panel) is modeled as a multi-component system consisting of an exponential bulge, think disk, thick disk, and extended spheroid. The model shown here has a surface mass density best modeled by a bulge-thin disk-thick disk system.

$a_{\mathrm{b}}<1 \mathrm{kpc}$, the thick disk scale length $R_{\mathrm{d}, \text { thick }}$ is larger than the thin disk scale length $R_{\mathrm{d}}$, and the spheroid scale length $a_{\mathrm{s}}>a_{\mathrm{b}}$. A simple $\chi^{2}$ estimate of the success of each model in reproducing the surface mass density profile of the remnant is used to determine the relative mass in bulge, disk, and spheroid components. When the model fits suggest that either a thick disk or an extended spheroid models provide comparable fits to the projected stellar distribution, the rotational support $V_{\text {rot }} / \sigma$ of the stars at large radii is used to select a preferred model. In this case, dispersion supported systems $\left(V_{\text {rot }} / \sigma<1\right)$ are assigned extended spheroids and rotationally-supported systems $\left(V_{\text {rot }} / \sigma \gtrsim 1\right)$ are assigned thick disk components. The best-fit surface mass density models are reported in Table 2] including the remnant bulge-disk-spheroid mass ratios. For comparison, Table 2 also lists the best fit surface mass density parameters for a $V_{\mathrm{vir}}=160 \mathrm{~km} \mathrm{~s}^{-1}$ progenitor (model ICs) with a gas fraction of $f_{\text {gas }}=0.4$ measured using the same method.

\section{RESULTS}

The suite of simulations provides an extensive set of remnants that characterize the ability of extremely gas-rich mergers to produce both disk components in spheroid-dominated systems and disk-dominated systems whose structure and kinematics closely resemble large spiral galaxies. In nearly every gas-rich merger we simulated (save model DCs, see below) the new stars formed after the peak of the SFR produced a rotationally-supported structure. These remnant disks are ubiquitous in gas-rich mergers, but the extent to which they dominate the stellar content and the degree of rotational support shows trends with the strength of pressurization in the ISM, the presence of growing supermassive BHs, progenitor gas fraction, orbit and disk orientation, and progenitor mass ratio. Below, we consider the impact of these merger properties in turn.

\subsection{Feedback Study}

The kinematic properties of the remnants exhibit significant quantitative differences as the pressure support of the ISM is varied. The stellar rotation curves of the 


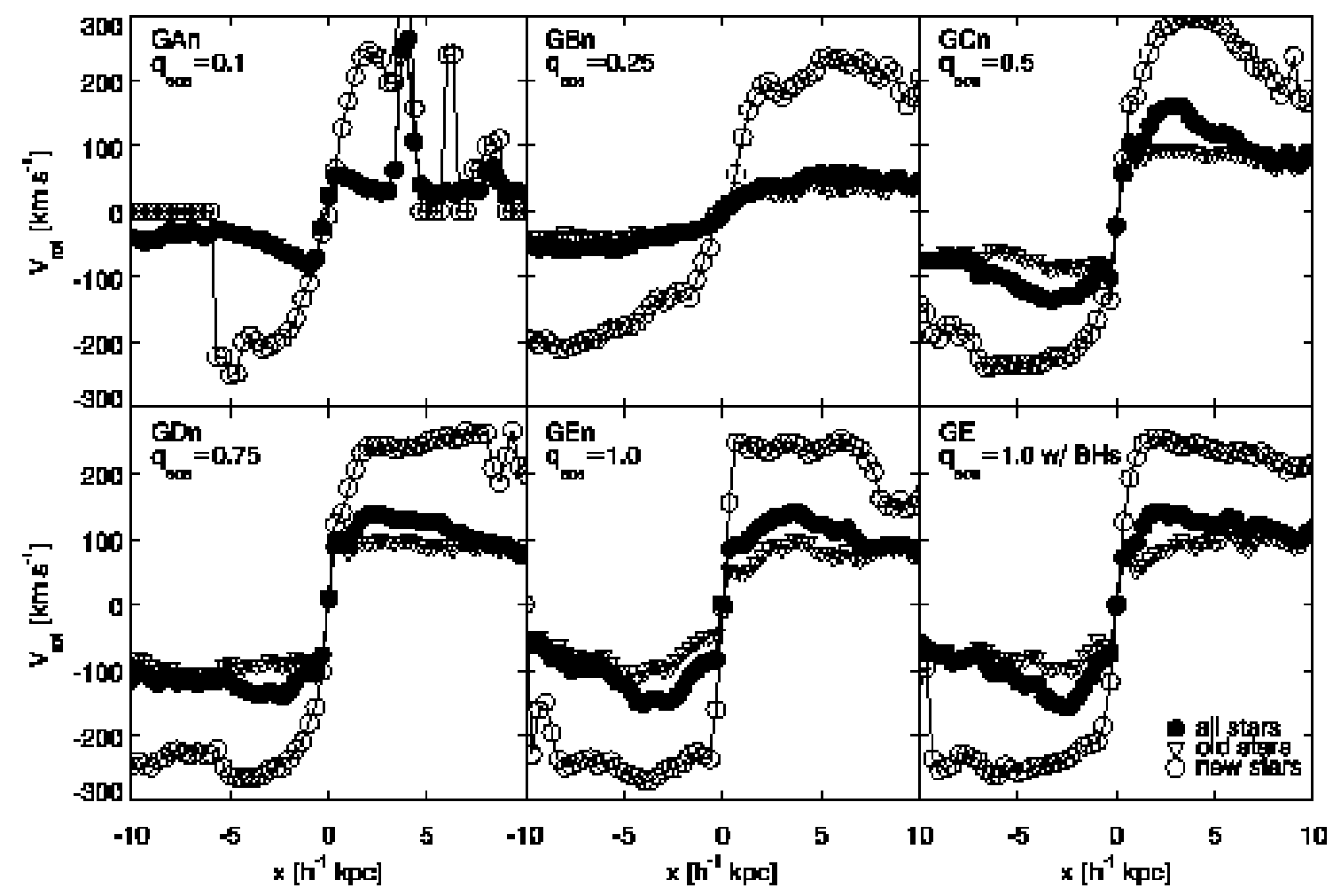

FIG. 3. - Stellar rotation curve of merger remnants, for models with differing ISM pressurization. Shown is the rotation of stars formed before the merger (open triangles), after the merger (open circles), and for all stars in the system (filled circles). The amount of rotation in the disk remnant correlates strongly with gas pressurization, increasing from the upper left panel $\left(q_{\mathrm{EOS}}=0.1\right.$, model GAn) to the bottom middle panel $\left(q_{\mathrm{EOS}}=1.0\right.$, model GEn). The nearly isothermal ISM model ( $q_{\mathrm{EOS}}=0.1$, model GAn) produces almost no net rotation in the stellar disk, while moderately to strongly pressurized models $\left(q_{\mathrm{EOS}}=0.5-1.0\right.$, models GCn-GEn) yield a rotating stellar component. The lower right panel shows a strongly pressurized ISM model $(q \mathrm{EOS}=1.0$, model GE) with BHs.

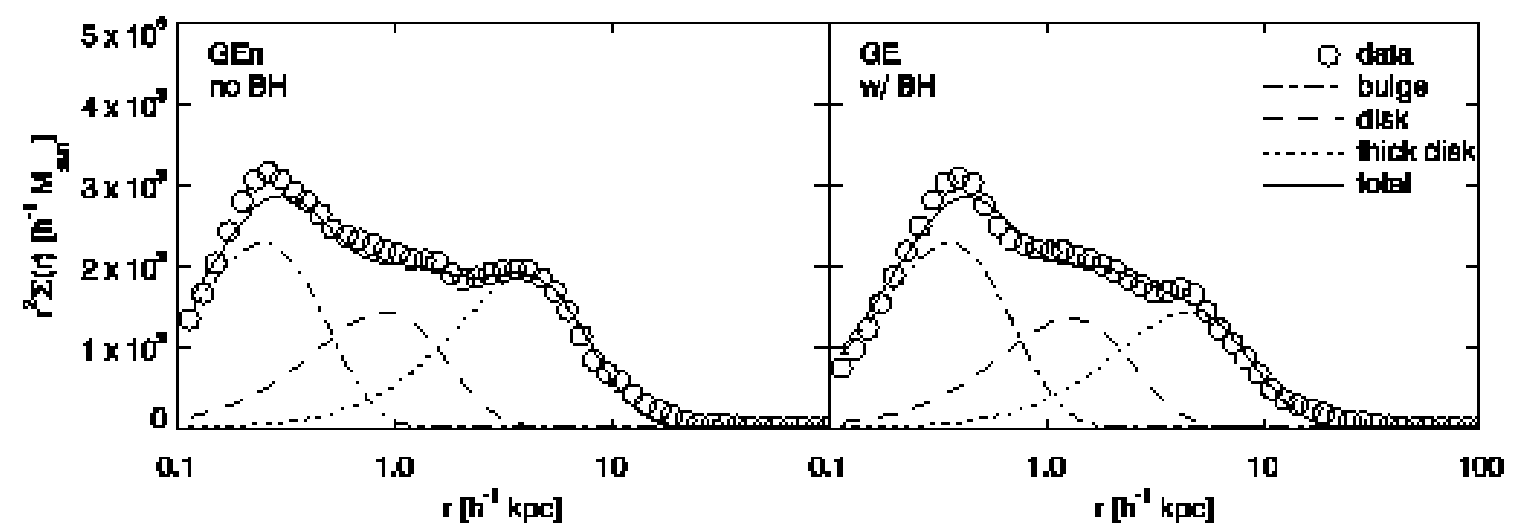

FIG. 4.- Remnant stellar mass surface density with a strongly pressurized ( $q_{\mathrm{EOS}}=1.5$ ) ISM, with (right panel, model GE) and without (left panel, model GEn) black hole feedback. Shown is the measured stellar surface density (open circles), and bulge (dot-dashed line), thin disk (dashed line), thick disk (dotted line), and composite (solid line) mass models fits. We fit exponential disks to the central bulge and stellar disk components and a de Vaucouleurs (1948) profile to the spheroid. As discussed by Robertson et al. (2006), black hole feedback reduces slightly reduces the mass of the central stellar spheroid and increases its effective radius. The relative sizes of the bulge and disk components are reported in Table 2

merger remnant in the $q_{\mathrm{EOS}}=0.1,0.25,0.5,0.75$, and 1.0 models without BHs are plotted in Figure 3 for a progenitor gas fraction of $f_{\text {gas }}=0.99$. The strength of the rotating stellar component correlates with the pressurization of the ISM. The least effective ISM model for producing a remnant disk is the weakly pressurized, nearly isothermal model $\left(q_{\mathrm{EOS}}=0.1\right.$, model GAn, upper left panel) that yields a slowly rotating, clumpy stellar rem- nant. The gaseous progenitor disks in model GAn are not stable against Toomre (1964) instability as the sound speed of the weakly pressurized gas cannot support the massive gas disk against self-gravity. The gaseous disk efficiently converts into stellar clumps before and during the collision, producing a remnant with very little average rotation $\left(\max V_{\text {rot }} \approx 90 \mathrm{~km} \mathrm{~s}^{-1}\right)$. However, the scant amount of gas remaining after the final coalescence 
Table 2. Merger Remnants

\begin{tabular}{|c|c|c|c|c|c|c|c|c|c|c|c|c|c|}
\hline Model & $\Sigma_{\mathrm{b}}$ & $a_{\mathrm{b}}$ & $\overline{\Sigma_{\mathrm{d}}}$ & $R_{\mathrm{d}}$ & $\overline{\Sigma_{\mathrm{s}}}$ & $a_{\mathrm{s}}$ & $\Sigma_{\mathrm{d}, \text { thick }}$ & $R_{\mathrm{d}, \text { thick }}$ & $M_{\mathrm{b}}$ & $M_{\mathrm{d}}$ & $M_{\mathrm{S}}$ & $M_{\mathrm{d}, \text { thick }}$ & B:D:S \\
\hline ICs & $\cdots$ & $\cdots$ & $4.9 \mathrm{e} 8$ & 2.73 & $\cdots$ & $\cdots$ & $\cdots$ & $\cdots$ & $\cdots$ & $2.3 \mathrm{e} 10$ & $\cdots$ & $\cdots$ & $0.0: 1.0: 0.0$ \\
\hline \multicolumn{14}{|c|}{$\mathrm{BHs}$} \\
\hline GA & $6.9 \mathrm{e} 10$ & 0.24 & $2.1 \mathrm{e} 8$ & 3.50 & $8.3 \mathrm{e} 7$ & 4.10 & $\cdots$ & $\cdots$ & $2.6 \mathrm{e} 10$ & $1.6 \mathrm{e} 10$ & $3.1 \mathrm{e} 10$ & $\cdots$ & $1.6: 1.0: 1.9$ \\
\hline GB & $3.9 \mathrm{e} 10$ & 0.41 & $2.1 \mathrm{e} 8$ & 4.48 & $\ldots$ & $\ldots$ & 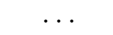 & $\ldots$ & $4.2 \mathrm{e} 10$ & $2.6 \mathrm{e} 10$ & & & $1.5: 1.0: 0.0$ \\
\hline GC & $2.0 \mathrm{e} 11$ & 0.17 & $1.6 \mathrm{e} 9$ & 1.55 & $\ldots$ & $\ldots$ & $3.3 \mathrm{e} 7$ & 5.19 & $3.8 \mathrm{e} 10$ & $2.5 \mathrm{e} 10$ & $\ldots$ & $5.7 \mathrm{e} 9$ & $1.2: 1.0: 0.0$ \\
\hline GD & $7.3 \mathrm{e} 10$ & 0.18 & $1.9 \mathrm{e} 9$ & 1.42 & $8.0 \mathrm{e} 7$ & 2.89 & $\ldots$ & 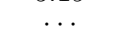 & $1.6 \mathrm{e} 10$ & $2.4 \mathrm{e} 10$ & $1.5 \mathrm{e} 10$ & & $1.0: 1.6: 1.0$ \\
\hline GE & $1.3 \mathrm{e} 11$ & 0.18 & $5.9 \mathrm{e} 9$ & 0.65 & $\ldots$ & $\ldots$ & $5.2 \mathrm{e} 8$ & 2.26 & $2.7 \mathrm{e} 10$ & $1.6 \mathrm{e} 10$ & $\ldots$ & $1.6 \mathrm{e} 10$ & $1.0: 1.2: 0.0$ \\
\hline \multicolumn{14}{|c|}{ No BHs } \\
\hline GAn & $4.3 \mathrm{e} 10$ & 0.28 & & & $1.6 \mathrm{e} 8$ & 3.88 & $\cdots$ & $\cdots$ & $2.1 \mathrm{e} 10$ & & $5.6 \mathrm{e} 10$ & $\cdots$ & $1.0: 0.0: 2.5$ \\
\hline GBn & $5.0 \mathrm{e} 10$ & 0.28 & $8.2 \mathrm{e} 7$ & 4.45 & $3.7 \mathrm{e} 8$ & 1.97 & $\ldots$ & $\ldots$ & $2.6 \mathrm{e} 10$ & $1.0 \mathrm{e} 10$ & $3.3 \mathrm{e} 10$ & $\ldots$ & $2.5: 1.0: 3.2$ \\
\hline GCn & $1.8 \mathrm{e} 11$ & 0.18 & $1.3 \mathrm{e} 9$ & 1.61 & $1.8 \mathrm{e} 7$ & 4.15 & $\ldots$ & $\ldots$ & $4.1 \mathrm{e} 10$ & $2.1 \mathrm{e} 10$ & 7.3e9 & $\cdots$ & $5.6: 2.8: 1.0$ \\
\hline GDn & $1.9 \mathrm{e} 11$ & 0.18 & $1.2 \mathrm{e} 9$ & 1.48 & $\ldots$ & $\ldots$ & $1.4 \mathrm{e} 8$ & 3.21 & $4.1 \mathrm{e} 10$ & $1.6 \mathrm{e} 10$ & $\cdots$ & $9.6 \mathrm{e} 9$ & $1.5: 1.0: 0.0$ \\
\hline GEn & $2.7 \mathrm{e} 11$ & 0.12 & $1.3 \mathrm{e} 10$ & 0.45 & $\ldots$ & $\ldots$ & $8.5 \mathrm{e} 8$ & 2.02 & $2.8 \mathrm{e} 10$ & $1.7 \mathrm{e} 10$ & $\ldots$ & $2.1 \mathrm{e} 10$ & $1.0: 1.4: 0.0$ \\
\hline \multicolumn{14}{|c|}{ Orbital Study } \\
\hline GCoC & $7.0 \mathrm{e} 10$ & 0.25 & $1.5 \mathrm{e} 9$ & 1.78 & & & $3.0 \mathrm{e} 7$ & 5.39 & $2.7 \mathrm{e} 10$ & $3.0 \mathrm{e} 10$ & & $5.5 \mathrm{e} 9$ & $1.0: 1.3: 0.0$ \\
\hline GCoE & $5.7 \mathrm{e} 10$ & 0.27 & $6.0 \mathrm{e} 8$ & 2.14 & $1.6 \mathrm{e} 8$ & 2.30 & $\ldots$ & $\ldots$ & $2.6 \mathrm{e} 10$ & $1.7 \mathrm{e} 10$ & $2.0 \mathrm{e} 10$ & $\ldots$ & $1.5: 1.0: 1.1$ \\
\hline GCoF & $4.7 \mathrm{e} 10$ & 0.25 & $1.7 \mathrm{e} 9$ & 1.96 & .. & $\ldots$ & & $\ldots$ & $1.9 \mathrm{e} 10$ & $4.2 \mathrm{e} 10$ & $\ldots$ & . & $1.0: 2.1: 0.0$ \\
\hline $\mathrm{GCoO}$ & $7.2 \mathrm{e} 10$ & 0.22 & $1.5 \mathrm{e} 9$ & 1.94 & $\cdots$ & $\cdots$ & $9.3 \mathrm{e} 6$ & 5.59 & $2.3 \mathrm{e} 10$ & $3.7 \mathrm{e} 10$ & $\cdots$ & $1.8 \mathrm{e} 9$ & $1.0: 1.6: 0.0$ \\
\hline GCoP & $6.2 \mathrm{e} 10$ & 0.24 & $1.6 \mathrm{e} 9$ & 1.43 & $9.0 \mathrm{e} 7$ & 2.90 & $\ldots$ & $\ldots$ & $2.3 \mathrm{e} 10$ & $2.1 \mathrm{e} 10$ & $1.7 \mathrm{e} 10$ & $\ldots$ & $1.3: 1.2: 1.0$ \\
\hline GCrA & $5.7 \mathrm{e} 10$ & 0.32 & $2.0 \mathrm{e} 9$ & 1.28 & $2.8 \mathrm{e} 7$ & 4.29 & $\ldots$ & $\cdots$ & $3.7 \mathrm{e} 10$ & $2.1 \mathrm{e} 10$ & $1.1 \mathrm{e} 10$ & $\cdots$ & $3.1: 1.8: 1.0$ \\
\hline GCrB & $2.4 \mathrm{e} 10$ & 0.35 & $8.6 \mathrm{e} 8$ & 2.04 & & & $6.1 \mathrm{e} 7$ & 6.02 & $1.9 \mathrm{e} 10$ & $2.2 \mathrm{e} 10$ & & $1.3 \mathrm{e} 10$ & $1.0: 1.8: 0.0$ \\
\hline GCrC & $4.0 \mathrm{e} 9$ & 0.54 & $1.5 \mathrm{e} 8$ & 3.32 & $1.0 \mathrm{e} 8$ & 4.31 & $\ldots$ & $\ldots$ & $7.5 \mathrm{e} 9$ & $1.0 \mathrm{e} 10$ & $4.4 \mathrm{e} 10$ & $\cdots$ & $1.0: 1.4: 5.9$ \\
\hline GCrD & $\cdots$ & 0.67 & $4.6 \mathrm{e} 7$ & 5.57 & $2.0 \mathrm{e} 8$ & 3.42 & $\cdots$ & $\cdots$ & $\cdots$ & $9.1 \mathrm{e} 9$ & $5.4 \mathrm{e} 10$ & $\cdots$ & $0.0: 1.0: 5.9$ \\
\hline \multicolumn{14}{|c|}{ Gas Fraction Study } \\
\hline$\overline{\mathrm{DC}}$ & $3.3 \mathrm{e} 10$ & 0.25 & $2.7 \mathrm{e} 7$ & 6.15 & $1.6 \mathrm{e} 8$ & 3.65 & & 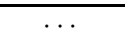 & $1.3 \mathrm{e} 10$ & $6.6 \mathrm{e} 9$ & $4.9 \mathrm{e} 10$ & $\bar{\cdots}$ & $2.0: 1.0: 7.5$ \\
\hline $\mathrm{EC}$ & $6.7 \mathrm{e} 10$ & 0.21 & $1.9 \mathrm{e} 7$ & 4.71 & $1.7 \mathrm{e} 8$ & 3.38 & .. & $\ldots$ & $2.0 \mathrm{e} 10$ & $2.7 \mathrm{e} 9$ & $4.6 \mathrm{e} 10$ & $\ldots$ & $7.6: 1.0: 17$ \\
\hline $\mathrm{FC}$ & $2.4 \mathrm{e} 11$ & 0.11 & $7.7 \mathrm{e} 9$ & 0.54 & $2.9 \mathrm{e} 7$ & 4.89 & $2.7 \mathrm{e} 8$ & 2.77 & $1.9 \mathrm{e} 10$ & $1.4 \mathrm{e} 10$ & $1.6 \mathrm{e} 10$ & $1.3 \mathrm{e} 10$ & $1.1: 1.7: 1.0$ \\
\hline \multicolumn{14}{|c|}{ Minor Merger Study } \\
\hline $\mathrm{DCm}$ & $4.3 \mathrm{e} 9$ & 0.57 & $3.7 \mathrm{e} 8$ & 3.36 & & & 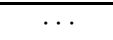 & 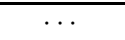 & $9.4 \mathrm{e} 9$ & $2.6 \mathrm{e} 10$ & & $\cdots$ & $1.0: 2.8: 0.0$ \\
\hline $\mathrm{ECm}$ & $3.6 \mathrm{e} 9$ & 0.58 & $3.3 \mathrm{e} 8$ & 3.14 & $1.3 \mathrm{e} 7$ & 4.70 & .. & $\ldots$ & $7.6 \mathrm{e} 9$ & $2.0 \mathrm{e} 10$ & $6.8 \mathrm{e} 9$ & $\cdots$ & $1.1: 3.0: 1.0$ \\
\hline $\mathrm{FCm}$ & $7.5 \mathrm{e} 9$ & 0.36 & $3.7 \mathrm{e} 8$ & 3.06 & $1.3 \mathrm{e} 7$ & 3.66 & $\cdots$ & $\cdots$ & $6.3 \mathrm{e} 9$ & $2.1 \mathrm{e} 10$ & $4.2 \mathrm{e} 9$ & $\cdots$ & $1.5: 5.1: 1.0$ \\
\hline \multicolumn{14}{|c|}{$V_{\text {vir }}=80 \mathrm{~km} \mathrm{~s}^{-1}$ Study } \\
\hline DCs & $8.7 \mathrm{e} 9$ & 0.12 & $1.0 \mathrm{e} 9$ & 0.33 & $6.7 \mathrm{e} 7$ & 2.15 & $\ldots$ & $\ldots$ & $8.5 \mathrm{e} 8$ & $7.2 \mathrm{e} 8$ & $7.1 \mathrm{e} 9$ & $\ldots$ & $1.1: 1.0: 9.8$ \\
\hline ECs & $3.3 \mathrm{e} 10$ & 0.10 & $9.1 \mathrm{e} 8$ & 0.46 & $3.5 \mathrm{e} 7$ & 2.43 & $\cdots$ & $\cdots$ & $2.1 \mathrm{e} 9$ & $1.2 \mathrm{e} 9$ & $4.8 \mathrm{e} 9$ & $\cdots$ & $1.7: 1.0: 3.9$ \\
\hline FCs & $3.2 \mathrm{e} 10$ & 0.10 & $1.5 \mathrm{e} 9$ & 0.34 & $5.0 \mathrm{e} 7$ & 1.92 & $\ldots$ & $\ldots$ & $2.0 \mathrm{e} 9$ & $1.2 \mathrm{e} 9$ & $4.2 \mathrm{e} 9$ & $\ldots$ & $1.7: 1.0: 3.4$ \\
\hline
\end{tabular}

in this merger still manages to produce a very small, rapidly rotating disk. Increasing the ISM pressurization to $q_{\mathrm{EOS}}=0.25$ (model GBn, upper middle panel) greatly reduces the presence of stellar clumps owing to instabilities and roughly doubles the radial extent of the rotating component, but does little to improve the average rotation of the remnant. Overall, low-pressurization ISM models do a poor job at producing remnants with substantial average rotation but can produce small, rapidly rotating remnant disk components.

Merger-driven disk formation will require a moderately to strongly pressurized ISM to provide enough kinematic stability to the gas to avoid forming too many stars before the final merger. The moderately to strongly pressurized models $\left(q_{\mathrm{EOS}}=0.5-1.0\right.$, GCn-GEn, upper left-lower middle panels) produce remnants that contain rapidly rotating stellar disks in addition to bulge or extended spheroid components. During the merger, the pressurization from the stiffer equation of state makes the ISM less compressible, reduces the central gas density, and leads to less conversion of gas into stars. A larger fraction of stars in the final remnant form after the height of the merger, increasing the mass of the disk component and the average rotational velocity of the stars. Just before the final coalescence, the moderately and strongly pressurized models have $f_{\text {gas }} \approx 0.5-0.6$, much larger than the $f_{\text {gas }} \approx 0.1$ in the $q_{\mathrm{EOS}}=0.1$ model and $f_{\text {gas }}=0.26$ in the $q_{\text {EOS }}=0.25$ model. The abundance of gas present during the final coalescence in the moderately and strongly pressurized models allows for stellar disks to form from gaseous remnant disks (e.g. Springel \& Hernquist 2005). Figure 4 shows the stellar surface mass density of two highly pressurized $\left(q_{\mathrm{EOS}}=1.0\right)$, gas-rich major merger models with (model GE, right panel) and without (model GEn, left panel) supermassive BH feedback. In both cases, stellar disks form from gaseous remnant disks created during the merger. The presence of supermassive BHs slightly decreases the mass of the central spheroid and increases its effective radius, in agreement with the findings of Robertson et al. (2006) for mergers that produce elliptical galaxies. Combined, the velocity fields shown in Figure 3 and the stel- 


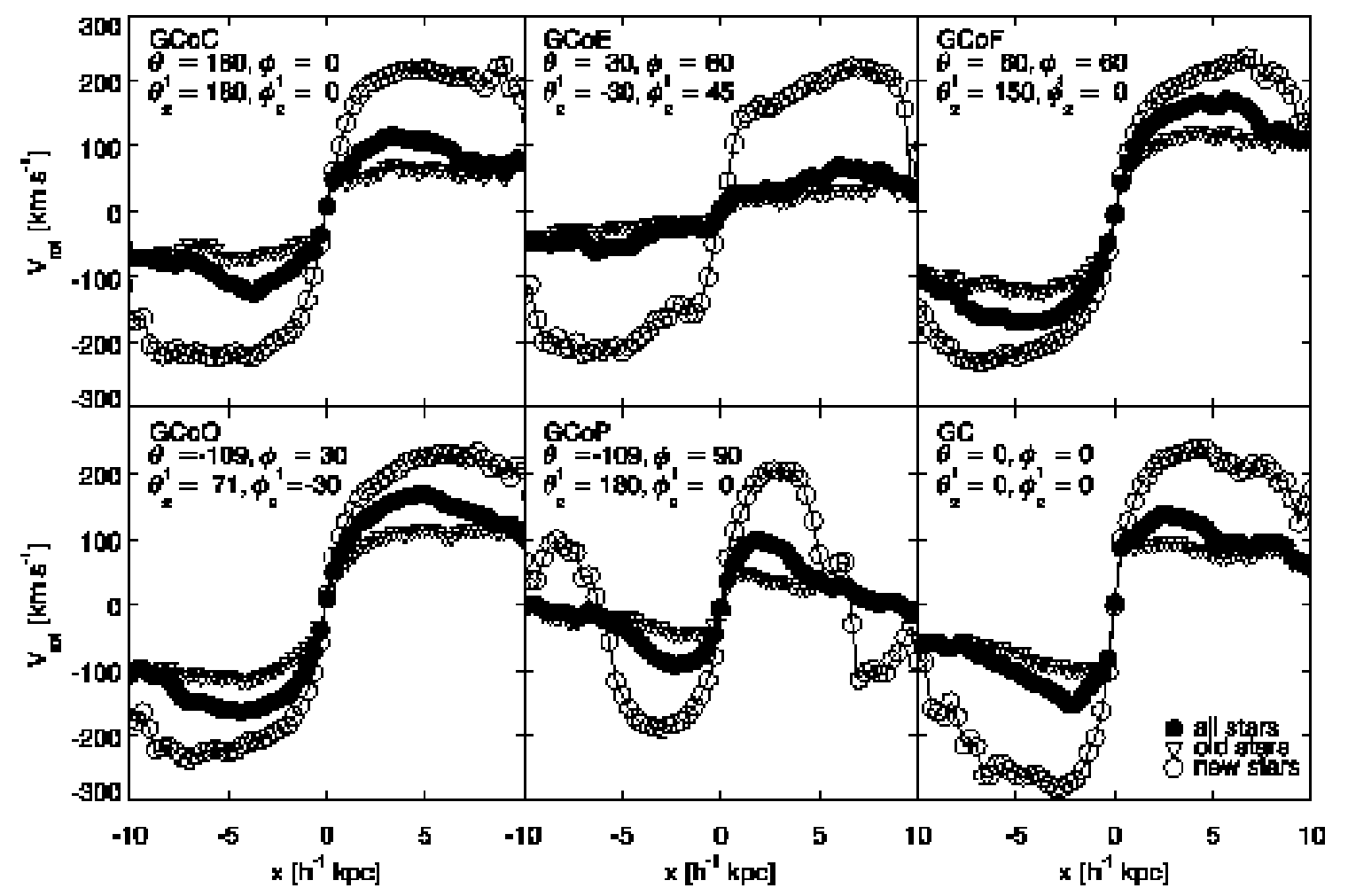

FIG. 5.- Rotation curves of remnants from mergers of galaxies with moderately pressurized ISM and growing supermassive black holes for a variety of disk orientations. Shown is the rotation for new stars formed after the merger (open circles), old stars formed before the merger (open triangles), and all stars (solid circles). The formation of rapidly rotating remnants is not limited to prograde-prograde coplanar mergers (model GC, lower right panel), but can occur in both polar (model GCoF, upper right panel) and inclined (model GCoO, lower left panel) orbits. Gas-rich mergers can also produce remnants with very little rotation (model GCoE, upper middle panel) or counter-rotating cores (model GCoP, lower middle panel).

lar disks shown in Figure 4 demonstrate that disk galaxy remnants containing rapidly rotating, stellar disk components can form from high-angular momentum, gas-rich mergers when the ISM is pressurized.

\subsection{Orbital Study}

While the results of 3.1 demonstrated that the pressurization of the multiphase ISM from star formation and feedback can produce remnant disks in gas-rich, prograde-prograde coplanar mergers, the generality of a merger-driven scenario for disk galaxy formation would require a wider range of encounters to produce remnant disks. Using simulations without star formation, Barnes (2002) demonstrated that gaseous disks could form in mergers that were not exactly coplanar, including polar orbits. Here, we examine the impact of varying a range of progenitor disk orientations and pericentric passage distances on remnant disk formation including the effects of star formation and energetic feedback mechanisms.

Figure [5] shows the rotation curves for six different fairly radial encounters, including a progradeprograde coplanar merger (model GC, lower right panel), retrograde-retrograde coplanar (model GCoC, upper left panel), polar (models GCoF, upper right panel, and GCoP, middle lower panel), and intermediate cases (models GCoE, upper middle panel, and GCoO, lower left panel). In each case, the same moderatelypressurized ISM $\left(q_{\mathrm{EOS}}=0.5\right)$ is used and enables the new stellar component to rapidly rotate. Remarkably, some highly non-coplanar encounters produce remnants whose entire stellar remnant is rapidly rotating (models $\mathrm{GCoF}$ and $\mathrm{GCoO}$ ) and demonstrate that merger-driven disk formation is not limited to the single coplanar orbit discussed by Springel \& Hernquist (2005). Certainly, some orbits lead to remnants with almost no average rotation (model GCoE), though with a rapidly rotating young stellar component. Interestingly, the retrograderetrograde polar encounter (model GCoP) produces a remnant with a new stellar component that counterrotates in its interior relative to the outer young stellar material. Previous studies have demonstrated the formation of counter-rotating disks to form in remnants of mergers involving progenitors with $f_{\text {gas }} \approx 0.1$ (Hernquist \& Barnes 1991) or in spiral galaxies via other mechanisms (e.g. Thakar \& Rvden 1996, 1998). We plan to examine the creation of counter-rotating cores through extremely gas-rich major mergers in subsequent work, but we note here that the relative ages of the older, central disk in model GCoP compared with its outer, younger disk is consistent with the picture for the formation of kinematically decoupled cores presented by Hernquist \& Barnes (1991) where the central disk forms primarily from strongly-torqued material during the first passage and the outer disk forms later from material that retains some of its original orbital or disk angular momentum. 


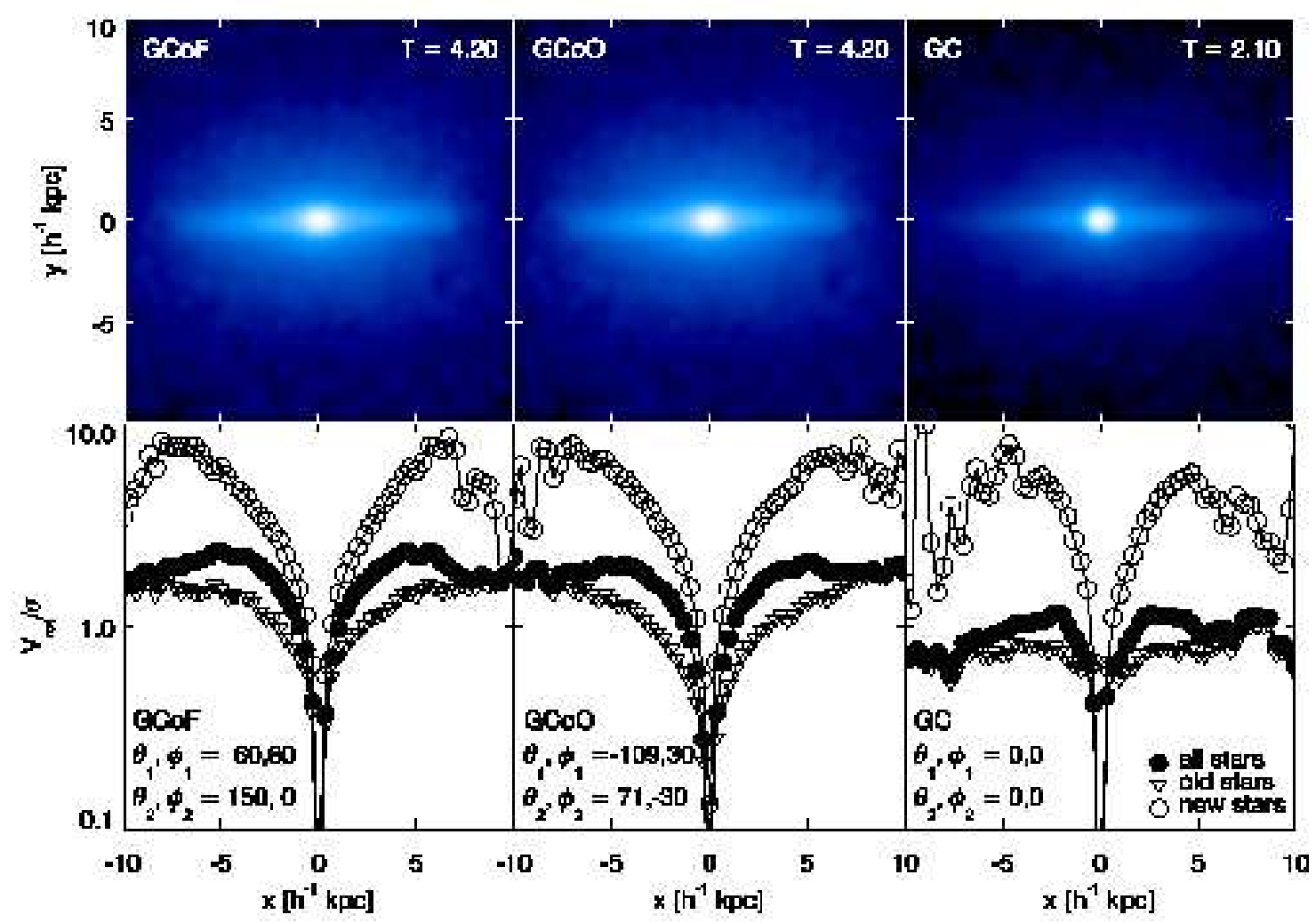

FIG. 6.- Rotational support $V_{\text {rot }} / \sigma$ and projected stellar mass for three orbits that produce remnant disks in very gas-rich mergers. Shown are the logarithmic surface density of the stars (upper panels) and the $V_{\text {rot }} / \sigma$ measured for all stars (solid circles), and stars formed before (open triangles) or after (open circles) the merger of galaxies with moderately pressurized ISM models $\left(q_{\mathrm{EOS}}=0.5\right)$. The polar $($ model GCoF, left panels) and inclined (model GCoO, middle panels) orbits lead to remnants that are disk-dominated and rotationally-supported. The prograde-prograde coplanar orbit (model GC, right panels) also produces a rotationally-supported disk system.

The rotational support of the remnants GCoF and GCoO formed from non-coplanar mergers, shown in Figure [6 with the coplanar encounter GC, is surprising but bolsters the merger-driven scenario for disk formation. In each merger, the total stellar rotational support exceeds $V_{\text {rot }} / \sigma=1$ and the new stellar component peaks at $V_{\text {rot }} / \sigma>5$. While the mass of the prograde-prograde coplanar merger remnant is still dominated by the central bulge (bulge-to-disk ratio $B: D \approx 1.2: 1.0$ ), the other models are disk-dominated with bulge-to-disk ratios of $B: D \approx 1.0: 2.1$ for model GCoF and $B: D \approx 1.0: 1.6$ for model $\mathrm{GCoO}$ including thick disk components. At the time of the merger these noncoplanar mergers have $f_{\text {gas }} \approx 0.6-0.7$ of their baryonic component in gas, compared with $f_{\text {gas }} \approx 0.5$ for the coplanar merger. The rotationally-supported remnants from non-coplanar orbits experience less star formation during their first passage than the coplanar orbit (see also Mihos \& Hernquist 1996), leaving more gas available to form a remnant disk after the final coalescence. The inclined merger model GCoE (Figure [5] upper middle panel) that produces a remnant with very little net rotation has a $f_{\text {gas }} \approx 0.2$ immediately after the final coalescence, whereas the disk remnants formed by models $\mathrm{GCoF}$ and $\mathrm{GCoO}$ have $f_{\text {gas }} \approx 0.5$ and $f_{\text {gas }} \approx 0.4$, respectively. Clearly, both the amount of gas present in the interacting system before the height of merger and the fraction of gas consumed during the final coalescence may influence the formation of remnant disks by improv- ing the rotational support of the remnant.

The simulations that explore the effects of disk orientation demonstrate that for a given amount of orbital angular momentum in a merger the contribution of disk angular momentum can affect the presence of a remnant disk, both by providing angular momentum to the remnant gaseous disk that forms the rapidly-rotating stellar component and by influencing the amount of gas consumed during the merger. However, the orbital configurations considered above only alter the disk orientation and not the orbital angular momentum of the encounter. Changing the pericentric passage distance can influence both the angular momentum of the remnant and the amount of gas available to form a remnant disk both by changing the timescale of the merger and by affecting the gas consumption during the interaction.

Figure 7 shows the rotation curves of remnants with moderately pressurized ISM models $\left(q_{\mathrm{EOS}}=0.5\right)$ formed in prograde-prograde coplanar gas-rich $\left(f_{\text {gas }}=0.99\right)$ mergers with pericentric passage distances increasing from $r_{\text {peri }}=R_{\text {d,progenitor }}=2.7 h^{-1} \mathrm{kpc}($ model GCrA, upper left panel) to $r_{\text {peri }}=0.3 R_{\text {vir.progenitor }}=48 h^{-1}$ kpc (model GCrD, lower right panel). Also shown for comparison is a polar merger with $r_{\text {peri }} \approx 2 R_{\mathrm{d} \text {,progenitor }}$ (upper right panel), the same pericentric passage distance as the prograde-prograde coplanar model GC (upper middle panel). The nearly head-on collision (model GCrA) produces a remnant with almost no net rotation; after the height of the merger, only a small amount of gas 


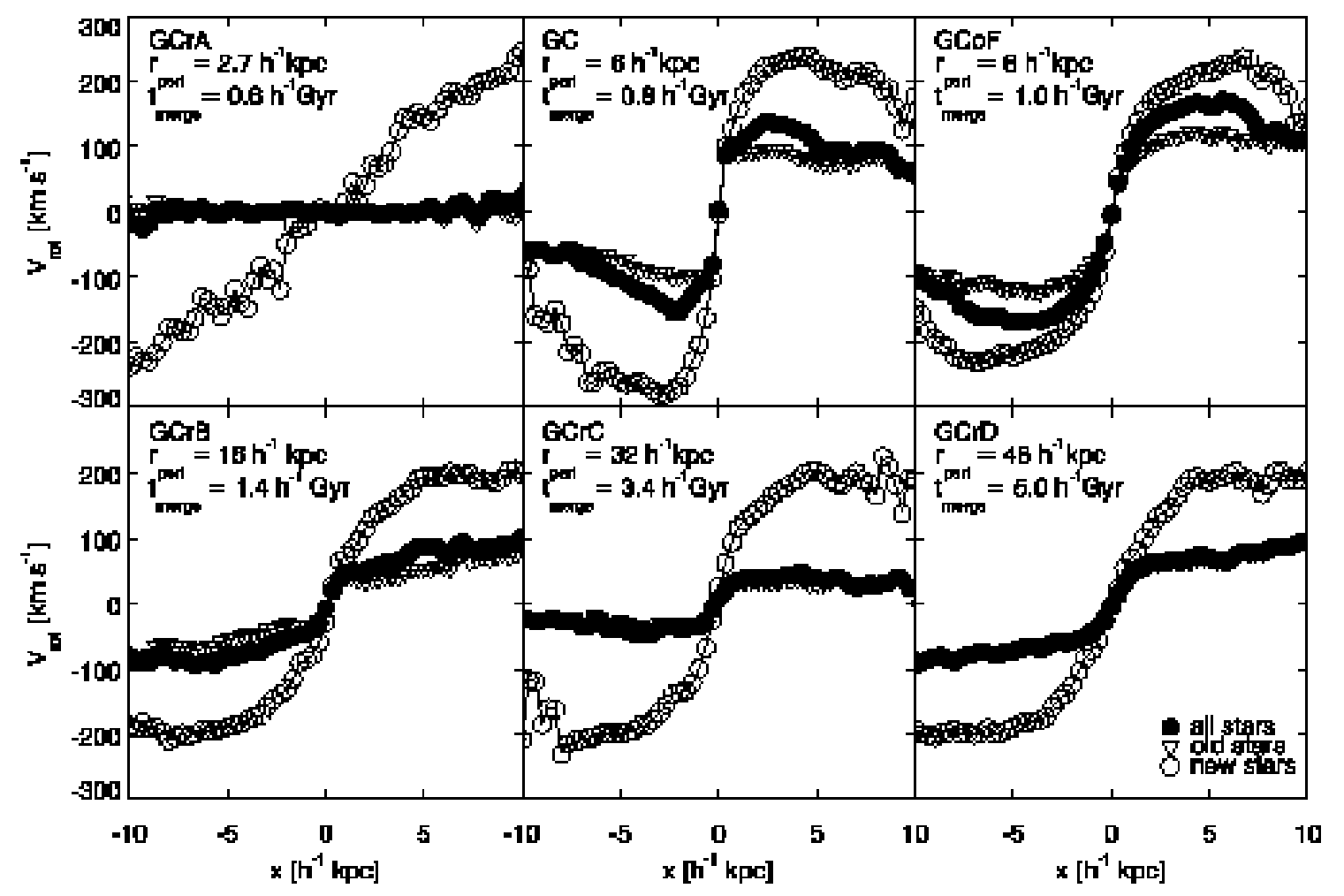

FIG. 7.- Rotation curves of remnants from mergers of galaxies with moderately pressurized ISM and growing supermassive black holes for a variety of pericentric passage distances. Shown is the rotation for new stars formed after the merger (open circles), old stars formed before the merger (open triangles), and all stars (solid circles) for pericentric passage distances increasing from $r_{\mathrm{peri}}=R_{\mathrm{d}}=2.7 h^{-1} \mathrm{kpc}$ (model GCrA, upper left panel) to $r_{\text {peri }}=0.3 R_{\mathrm{vir}}=48 h^{-1} \mathrm{kpc}$ (model GCrD, lower right panel). The formation of rapidly rotating remnants is appears to require orbits that are not too radial, since the first passage is violent and too much gas is converted to stars before the merger. Orbits also cannot be too wide, since the merger timescale is extended and results in a mostly stellar final merger. Remnants from mergers with pericentric passage distances of a few progenitor disk scale lengths, such as the coplanar (model GC, upper middle panel) and polar orbits shown here (model GCoF, upper right panel), appear to be most efficient at producing remnant disks.

remains $\left(f_{\text {gas }}=0.13\right)$. The gas available for a remnant disk increases to $f_{\text {gas }} \approx 0.5-0.6$ when the pericentric passage disk scale length is roughly twice the disk scale length (models $\mathrm{GC}$ and $\mathrm{GCoF}$ ), and decreases towards larger $r_{\text {peri }}$ with $f_{\text {gas }} \approx 0.2-0.4$ (models GCrB-GCrD). These orbits have a substantially longer merger timescale than the more radial orbits, allowing for more quiescent star formation and a larger stellar component present in the progenitors at the height of the merger. The competing effects of increasing the available orbital angular momentum by increasing $r_{\text {peri }}$ and decreasing the gas available to form a remnant disk owing to extended quiescent star formation then lead to a range of $r_{\text {peri }}$ from which remnant disks are likely to form. While additional simulations will be needed to better characterize the best orbits for remnant disk formation, from the simulations performed here we estimate that fairly radial orbits with pericentric passage distances a few times larger than the disk scale length are most ideal for merger-driven disk formation in gas-rich mergers.

\subsection{Gas Fraction Study}

The preceding feedback and orbital studies have demonstrated that gas-rich mergers with a pressurized ISM can produce remnant disks for a variety of orbital configurations. The results of those simulations suggest that the primary influence on the presence of remnant disks is the amount of gaseous material left after the final coalescence; increased feedback or favorable (though diverse) orbits reduce the SFR during the first pericentric passage and the height of the merger, leaving enough gas after the merger to form a rapidly-rotating stellar component. By altering directly the gas fraction of the progenitor systems, the role of gas fraction on the rotational support of merger remnants can be determined.

Figure 8 shows the impact of increasing the gas fraction from $f_{\text {gas }}=0.4$ (left panel, model DC) to $f_{\text {gas }}=0.8$ (right panel, model FC) in the progenitor disks. Shown are prograde-prograde coplanar mergers with a moderately pressurized ISM model $\left(q_{\mathrm{EOS}}=0.5\right)$. While the new stars in each model produce a rotationally-supported structure, the average rotational support of the system is well correlated with the gas fraction as judged from the increasing $V_{\text {rot }} / \sigma$ as a function of progenitor $f_{\text {gas }}$. The gas fraction of the merging galaxies before and after the final coalescence are nearly in proportion to the original gas progenitor gas fractions, with $f_{\text {gas }}=0.2$ before and $f_{\text {gas }}=0.1$ after the final merger in model DC and roughly twice that those values for the FC model with double the original gas content. However, the original $f_{\text {gas }}=0.8$ progenitors in the model FC merger are not gas-rich enough to produce a remnant that has a rotationally- 


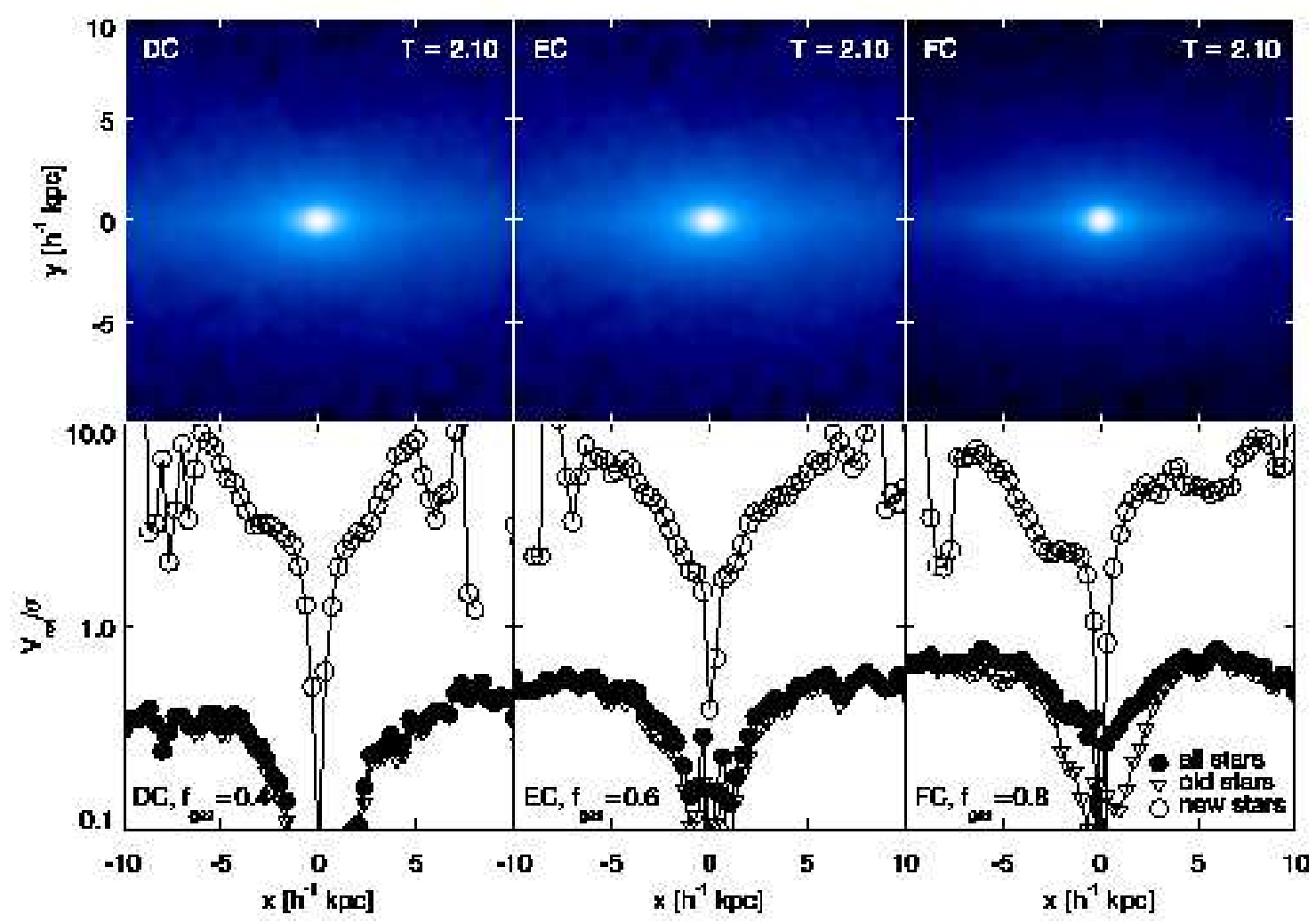

FIG. 8. - Rotational support $V_{\text {rot }} / \sigma$ and projected stellar mass for mergers with progenitor gas fractions ranging from $f_{\text {gas }}=0.4$ (model $\mathrm{DC}$, left panel) to $f_{\text {gas }}=0.8$ (model FC, right panel). mergers. Shown are the logarithmic surface density of the stars (upper panels) and the $V_{\text {rot }} / \sigma$ measured for all stars (solid circles), and stars formed before (open triangles) or after (open circles) the merger of galaxies with moderately pressurized ISM models $\left(q_{\mathrm{EOS}}=0.5\right)$. The rotational component of these dispersion dominated galaxies correlate with the gas fraction, and progenitor galaxies will likely need an initial gas fraction of $f_{\text {gas }}>0.8$ to produce remnant disks (see Figure 3 .

supported total stellar component with this orbital configuration. These simulations suggest that given the typical Gyr timescales involved, major merger progenitors must have original gas fractions $f_{\text {gas }} \gtrsim 0.8$ in order to satisfy the $f_{\text {gas }} \gtrsim 0.5$ requirement during final coalescence for a disk-dominated remnant.

\subsection{Minor Merger Study}

The simulations of 3.13 .3 have demonstrated that gas-rich mergers can lead to remnant disk systems with substantial rotational support. However, these simulations have modeled only equal mass encounters. While major mergers on average contribute significantly to the final mass of an average galaxy at the present day, minor mergers between unequal mass galaxies are cosmologically more frequent (Lacev \& Cole 1993).

The survival of disks in minor merger or infall scenarios have been readily addressed both semianalytically (Toth \& Ostriker 1992; Benson et al. 2004) and numerically (Quinn et al.|1993; Walker et al. 1996; Velazquez \& White 1999; Font et al. 2001; Barnes 2002). Recently, Bournaud et al. (2005) has simulated comparatively gas-poor $\left(f_{\text {gas }}<0.2\right)$ mergers between galaxies with mass ratios in the range $4: 1-10: 1$ which produce remnants with disk-like stellar structures but elliptical-like kinematics (see also Bournaud et al. 2004). Currently, the combined results of these analyses suggest that stellar minor mergers with disks will produce remnant systems that are kinematically dispersion- dominated but the cosmological infall of satellites expected in the $\Lambda \mathrm{CDM}$ cosmology is consistent with the observed distribution of thicknesses for disk galaxies.

Of additional interest to a scenario for merger-driven disk formation, Barnes (2002) demonstrated in hydrodynamical simulations without star formation that gas-rich minor mergers can produce small gaseous disk remnants. A cosmological scenario for merger-driven disk formation would likely involve such minor mergers given their abundance in the mass-accretion histories of galaxies. Figure 9 shows the rotational support of remnants formed in prograde-prograde coplanar mergers of the $V_{\text {vir }}=160$ $\mathrm{km} \mathrm{s}^{-1}$ galaxy model considered in 3.13 .3 with the $V_{\text {vir }}=80 \mathrm{~km} \mathrm{~s}^{-1}$ galaxy described in $\$ 2.4$ These minor mergers with mass ratio $m_{2}: m_{1} \approx 8: 1$ use a moderately pressurized ISM $\left(q_{\mathrm{EOS}}=0.5\right)$ and progenitor gas fractions increasing from $f_{\text {gas }}=0.4$ (model DCm, left panel) to $f_{\text {gas }}=0.8$ (model FCm, right panel). The remnants are disk-dominated and rotationally-supported, with $V_{\text {rot }} / \sigma \approx 2$ at $5 h^{-1} \mathrm{kpc}$. The smaller system is still massive enough to foment central star formation by driving gas inwards in the larger galaxy, increasing the velocity dispersion by a factor of $\approx 2$ to $\sigma \approx 80 \mathrm{~km} \mathrm{~s}^{-1}$ at the center each remnant. These results are qualitatively consistent with previous work simulating the response of galaxy disks to minor mergers at lower gas fractions $\left(f_{\text {cas }} \approx 0.1\right.$, e.g. Hernquist 1989; Mihos \& Hernquist 1994b; Hernquist \& Mihos 1995), but in our substantially more gas-rich systems the additional supply of gas 


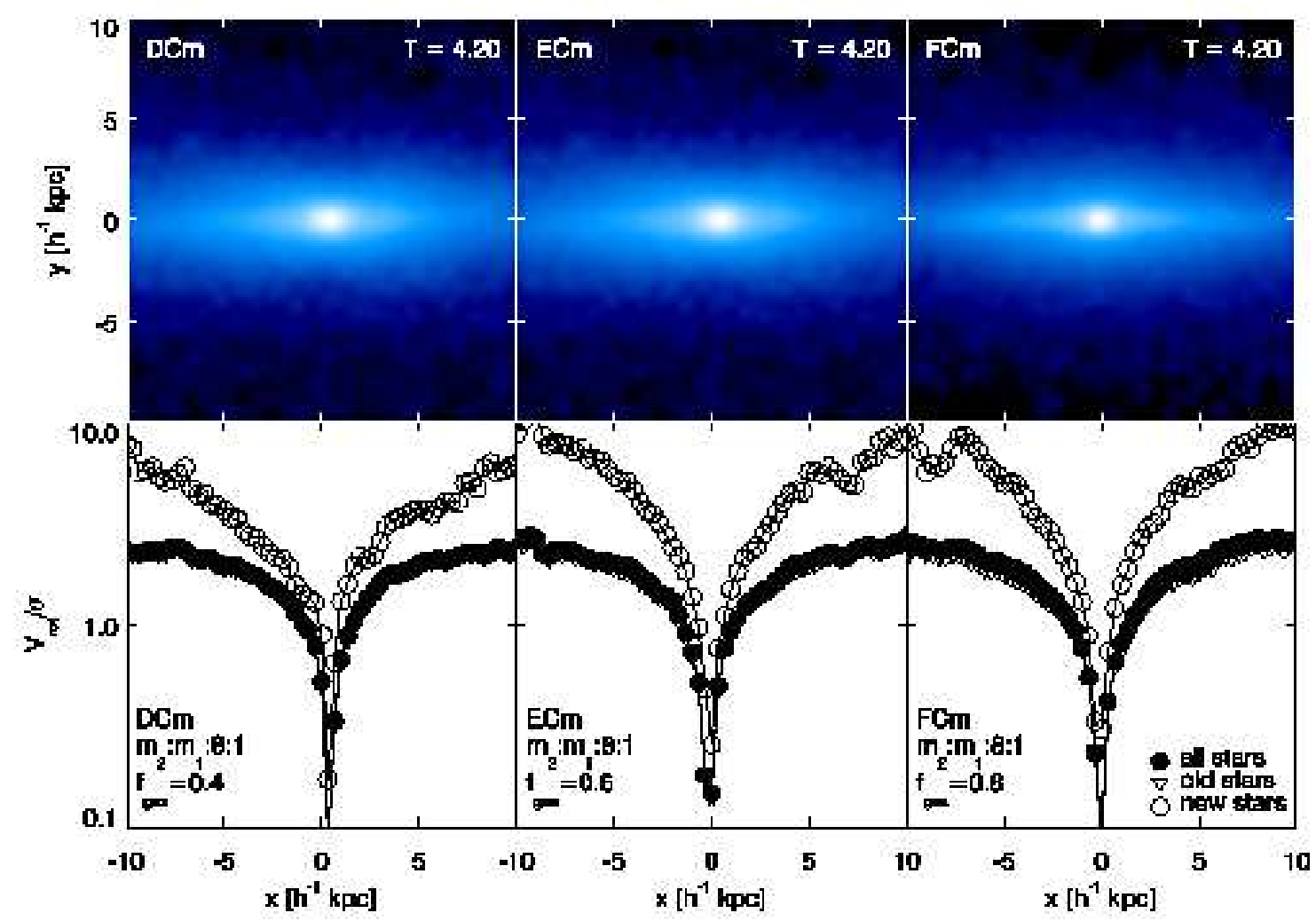

Fig. 9. - Rotational support $V_{\text {rot }} / \sigma$ and projected stellar mass for three minor mergers (mass ratio $m_{2}: m_{1}=8: 1$ ) for progenitor gas fractions ranging from $f_{\text {gas }}=0.4$ (model DCm, left panel) to $f_{\text {gas }}=0.8$ (model FCm, right panel). Shown are the logarithmic surface density of the stars (upper panels) and the $V_{\text {rot }} / \sigma$ measured for all stars (solid circles), and stars formed before (open triangles) or after (open circles) the merger of galaxies with moderately pressurized ISM models $\left(q_{\mathrm{EOS}}=0.5\right)$. The minor mergers produce disk-dominated, rapidly-rotating remnants with $V_{\mathrm{rot}} / \sigma>2$. The central velocity dispersion of the larger galaxy roughly double during the merger, but the rotational support of the remnants are considerably larger than the $V_{\text {rot }} / \sigma$ measured for comparable gas-poor minor mergers (e.g. Bournaud et al. 2005).

allows the larger galaxy to retain comparatively more mass-weighted stellar rotation by the end of the simulation through continued star formation in the extended gaseous disk. The velocity dispersion in the outer regions of our simulated remnants increases from $\sigma \approx 30$ $\mathrm{km} \mathrm{s}^{-1}$ in the larger progenitor at $5 h^{-1} \mathrm{kpc}$ before the merger to $\sigma \approx 50-60 \mathrm{~km} \mathrm{~s}^{-1}$ in the remnant disk, decreasing with progenitor gas fraction. While the remnant properties are not strong functions of the gas fraction for our very gas-rich mergers, with each remnant displaying similar rotational support and bulge-to-disk ratios $\left(B: D \approx 1: 2-1: 3\right.$, see Table 2), the $V_{\text {rot }} / \sigma \approx 2-2.5$ throughout most of the remnant disks substantially exceeds the $V_{\text {rot }} / \sigma \approx 1.6$ for the comparatively gas-poor mergers reported by Bournaud et al. (2005) for mergers of mass ratio $m_{2}: m_{1}=8: 1$ (see their Figure 10). We therefore infer that increasing the gas fraction of the disk progenitors of minor mergers beyond the maximum $f_{\text {gas }}=0.2$ used by Bournaud et al. (2005) will improve the rotational support of remnant disk galaxies, but we cannot fully detail the strength of the effect with the simulations presented here. However, based upon the success of the gas-rich minor mergers in retaining significant rotational support for the remnant disks we suggest that gas-rich minor mergers may be an arbiter of mergerdriven disk formation and should be studied further.

\subsection{Small Progenitor Study}

The scale-dependent physics of gas cooling and star formation may influence merger-driven disk formation for a mass-sequence of galaxies. To examine this possibility, we repeat the gas fraction study presented in 3.3 substituting smaller, $V_{\text {vir }}=80$ galaxies for the progenitor systems while maintaining the same orbit (i.e. the same physical $r_{\text {peri }}$ and $\left.r_{\text {init }}\right)$. Figure [10 shows the $V_{\text {rot }} / \sigma$ as a function of radius for mergers with moderately pressurized ISM models $\left(q_{\mathrm{EOS}}=0.5\right)$ and progenitor gas fractions increasing from $f_{\text {gas }}=0.4$ (model DCs, left panel) to $f_{\text {gas }}=0.8$ (model FCs, right panel). The remnants produced in these lower-mass mergers are rotationally-supported only in their outer regions. The merger with $f_{\text {gas }}=0.4$ progenitors (model DCs) does not contain a significant rotating component comprised from new stars, the only such system in our suite of simulations. The higher gas fraction progenitors (models ECs and FCs) do produce rotating structures from their young stellar components, but the average rotation of these systems is dominated by rotation in their large spheroid components.

As discovered for more massive progenitors in 33.3 the gas fractions of the merging systems both before and after the final coalescence scale roughly with the initial progenitor gas fraction for the same orbit and ISM pressurization. These systems each have gas fractions $f_{\text {gas }}<0.5$ 


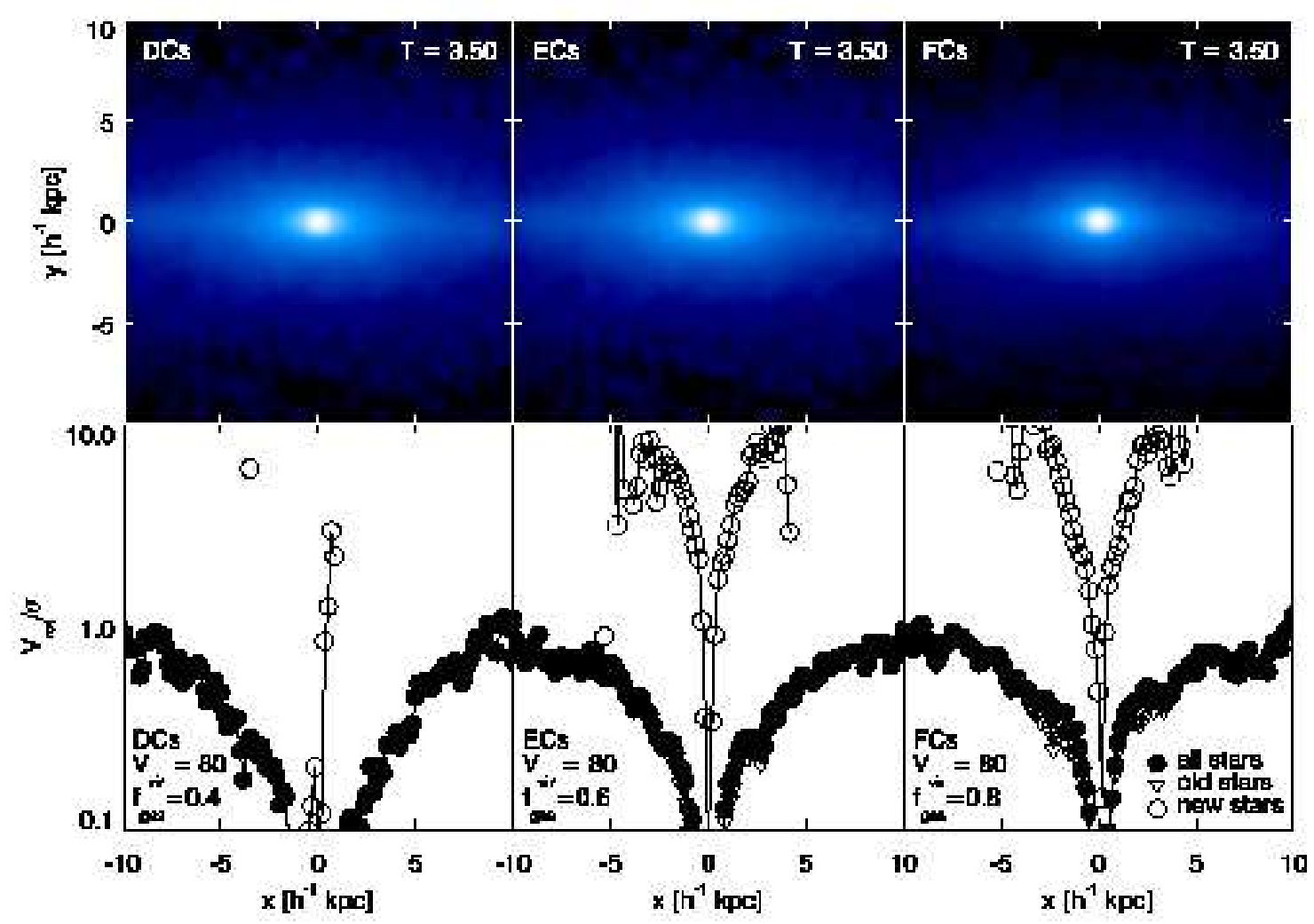

FIG. 10.- Rotational support $V_{\text {rot }} / \sigma$ and projected stellar mass for mergers with progenitor gas fractions ranging from $f_{\text {gas }}=0.4$ (model DC, left panel) to $f_{\text {gas }}=0.8$ (model FC, right panel). mergers for small $V_{\text {vir }}=80 \mathrm{~km} \mathrm{~s}^{-1}$ galaxies. Shown are the logarithmic surface density of the stars (upper panels) and the $V_{\text {rot }} / \sigma$ measured for all stars (solid circles), and stars formed before (open triangles) or after (open circles) the merger of galaxies with moderately pressurized ISM models $\left(q_{\mathrm{EOS}}=0.5\right)$. The rotational component of these dispersion dominated galaxies correlate with the gas fraction, though the relative importance of rotation is higher in these small mass systems than the more massive systems in similar mergers (see Figure 8). The progenitor galaxies will likely need an initial gas fraction of $f_{\text {gas }}>0.8$ and the merging system will need $f_{\text {gas }}>0.5$ just prior to the final coalescence to produce remnant disks (see Figure 3 .

just before the final coalescence and $f_{\text {gas }}<0.25$ immediately afterwards, below the gas fractions measured in mergers that produce remnant disks. While the gas densities in the disks of these systems are lower than in the more massive systems considered in $\$ 3.3$ and therefore produce relatively less star formation in quiescence, the merger timescale for the less massive systems is longer and leads to similar gas fractions at the final merger. If instead of using a physically identical orbit for both the lower-mass and higher-mass progenitors the orbits for the lower-mass progenitors were scaled with the disk scale lengths, or similarly the virial radii, the less massive systems would have merged on a shorter timescale and may have produced more rotationally-supported remnants. Given the increased rotational support in the remnants of lower-mass progenitors relative to the higher-mass progenitors, we speculate here that lower-mass systems may be more conducive to merger-driven disk formation than can be inferred from the simulations we have performed. We leave explorations of these suspicions to future work.

\section{DISCUSSION}

The suite of mergers simulated in this work indicates that merger-driven disk formation may viable in the gasrich mergers that build high-redshift galaxies. The requirements that the merging system is gas-dominated $\left(f_{\text {gas }} \gtrsim 0.5\right)$ during the final coalescence to provide enough gas immediately after the merger to sustain a remnant disk is likely a necessary but not sufficient condition for merger-driven disk formation. However, physical effects that increase the gas fraction during the final merger also improve the structure and rotational support of remnant disks. Increased pressurization of the ISM, favorable orbits with less-violent first passages or shorter merger timescales, small mass ratio mergers that decrease the interaction-induced star formation in the more massive progenitors, and simply increased gas fractions in the progenitors all improve the rotational support of the remnants and in some cases lead to diskdominated, rapidly rotating stellar remnants.

The importance of gas-rich mergers for a variety of galaxy properties has been increasingly realized (for a discussion see e.g., Brook et al. 2005), and the results of our simulations of gas-rich progenitors bear on hierarchical models for the mass assembly of disk galaxies. Abadi et al. (2003b) report that a substantial fraction of the thick disk stars of a simulated disk galaxy formed in a cosmological setting originate as tidal debris from satellite systems accreted after the thin disk forms. Brook et al. (2004, 2005, 2006) present a different scenario where the thick disk forms from multiple early gas-rich mergers $\left(f_{\text {gas }} \approx 0.5 ; \mathrm{C}\right.$. Brook, private communication) and the thin disk forms later from the gaseous debris of these accretion events. A recent photometric 
survey of the thick and thin components of edge-on disk galaxies by Yoachim \& Dalcanton (2006) and kinematic decompositions of the thick and thin disk components of two galaxies by Yoachim \& Dalcanton (2005) suggest that thick disks may be kinematically disjoint, with lower rotational velocities than galactic thin disks. This observational evidence supports the thick disk accretion scenario forwarded by Abadi et al. (2003b). However, if substantial thick disk mass accreted after the thin disk becomes predominately stellar then heating of the thin disk will occur anyway (e.g. Quinn et al. 1993). Instead, if the epoch of thick disk accretion occurs when the large thin disk is gas-rich, then our simulations of minor mergers suggest that the thick disk may be safely accreted without unreasonably increasing the thin disk velocity dispersion.

Our study of the formation of remnant disks in gas rich mergers provides a compliment to studies of elliptical galaxy formation in gas-rich merging (e.g. Robertson et al. 2006). Major mergers between disk galaxies with progenitor gas fractions $f_{\text {gas }} \leq 0.8$ almost uniformly produce spheroid-dominated systems as the systems are not gas-dominated at the time of final coalescence. Such spheroid-dominated remnants are similar to our lower gas fraction models EC-FC. As Robertson et al. (2006) demonstrate, these spheroid dominated systems obey elliptical galaxy scaling relations such as the Fundamental Plane (Diorgovski \& Davis 1987; Dressler et al. 1987).

Moreover, other apparently separate considerations make the case that if ellipticals did form through mergers, the progenitors must have been gas-rich (though not gas-dominated as the simulations here show that these events may leave behind systems with large disks). For example, Hernquist et al. (1993) showed that gas fractions larger than or of order $20-30 \%$ are required if mergers between disk galaxies are to account for the relatively high phase space densities of local ellipticals. Moreover, this same criterion ensures that the remnants obey observed scaling relations (e.g. Robertson et al. 2006) and match kinematic properties (e.g. Cox et al. 2006) of ellipticals.

The even more gas-rich mergers with progenitor gas fractions $f_{\text {gas }}>0.8$ with disk-dominated stellar components will not satisfy these elliptical galaxy scaling relations owing to their rotational support. These gasrich merging scenarios for elliptical galaxy formation and remnant disk galaxy formation are fully consistent, as the remnant stellar disks form in systems with gas fractions large enough to sustain remnant gaseous disks whereas elliptical galaxies are generated in highly dissipative events where the vast majority of the gas is consumed and a large gaseous remnant disk cannot form owing to the nearly complete depletion of gas. In gasrich merger events that lead to elliptical galaxy formation, almost all of the stars form before or during the final coalescence of the galaxies. The production of remnant disks in mergers requires that progenitors are comparably even more gas-rich to enable the formation of a remnant gaseous disk massive enough to eventually form a rapidly-rotating stellar disk that can dominate the stellar structure of the remnant. Naturally, this requires that the gas fraction before the final coalescence is $f_{\text {gas }}>0.5$, in agreement with the results of this study.
Effects that reduce the consumption of gas before the final coalescence assuage the merger-driven production of disks, and while our work concentrates on feedback and orbital effects as well as the gas fraction of the progenitors we note that additional structural properties of the progenitors that reduce gas consumption during the merger (e.g. bulges) may also play a role in the formation of remnant disks. Lastly, the range of Hubble types observed for spiral galaxies spans a considerable range in both rotational support $\left(V_{\mathrm{rot}} / \sigma\right)$ and bulge-todisk ratio. The merger-driven scenario for disk galaxy formation here may account for systems with bulge components but likely cannot explain very late-type systems (e.g. Sd galaxies), which presumably must form almost entirely through dissipative processes that do not produce hot stellar components.

We emphasize that feedback associated with black hole growth, while essential for accounting for the observed properties of quasars (Hopkins et al. 2005d a b) and the red colors of ellipticals (e.g. Springel et al. 2005a; Hopkins et al. 2005d), has little impact on the global structural properties of the remnants studied here. This is consistent with the work of Robertson et al. (2006) and Cox et al. (2006) who found that black hole feedback likewise has negligible consequence for ellipticals on scales of order the effective radius. The signatures of black hole growth on the stellar distribution of merger remnants thus appears subtle and may be restricted to modifying the characteristics of the central starburst population formed during the merger (e.g. Mihos \& Hernquist 1994a d).

\section{SUMMARY}

To address the often destructive impact of the frequent merging in the $\Lambda \mathrm{CDM}$ cosmology on spiral galaxies, we propose a new, merger-driven scenario for disk galaxy formation at high redshifts that supplements the standard picture based on dissipational collapse (White \& Rees 1978; Blumenthal et al. 1984). In this scenario, gas-rich mergers can form rotationallysupported gaseous structures from residual angular momentum after the final coalescence. This rapidly-rotating material will form stars, and if enough material is available to form a substantial rotationally-supported component the remnant can resemble a disk galaxy both structurally and kinematically. We perform a suite of twenty-eight merger simulations to address the feasibility of such a scenario for disk galaxy formation and demonstrate that remnant disks can form from sufficiently gas rich mergers with a variety of orbits, mass ratios, and physical models for the ISM or the inclusion of growing supermassive black holes. We provide a detailed summary of these results below.

- We verify the results of by Barnes (2002) and Springel \& Hernquist (2005) in demonstrating that gas-rich mergers can produce remnant disks. Nearly every gas-rich merger produces a rapidlyrotating structure from stars formed in remnant gaseous disks after the merger. Furthermore, we demonstrate that certain gas-rich mergers can produce remnants whose entire stellar structure is rotationally-supported. A necessary condition for the formation of these disk-dominated remnants is 
inferred to be a gas fraction of $f_{\text {gas }}>0.5$ just prior to the final coalescence, which we have referred to as being "gas-dominated". Merger-driven disk formation is shown to occur under a variety of gasdominated mergers and is not limited to e.g. idealized prograde-prograde coplanar mergers. Favorable equal mass encounters for merger-driven disk formation include certain polar and inclined orbits with limited star formation during the first pericentric passage. A successful merger-driven disk formation scenario would requires extremely gasrich progenitors with gas fractions $f_{\text {gas }} \geq 0.8$, and is therefore likely limited to high redshift galaxy assembly.

- The rotational support of remnants is shown to correlate with the pressurization of the ISM. Using the multiphase ISM model of Springel \& Hernquist (2003) modified to allow for an adjustable pressurization in the form of an effective equation of state (Springel et al. 2005b), the merger simulations demonstrate that pressurized ISM models limit the amount of gas consumed before the final coalescence of the progenitors. The reduced star formation increases the gaseous material available to form a rapidly-rotating remnant disk and improves the rotational support of the remnant system. Weakly pressurized ISM models either produce unstable progenitors owing to the high gas fractions or have efficient star formation. In both cases the merging systems experience a mostly stellar collision that simply kinematically heats the existing stars without producing a newly-formed disk to contribute to the rotation of the remnant.

- Gas-rich minor mergers can produce disk galaxies with substantial rotational support $\left(V_{\text {rot }} / \sigma>2\right)$.
The mass ratio $m_{2}: m_{1}=8: 1$ merger simulated for a range of gas fractions produces a remnant disk galaxy rotationally-supported at all measured radii $r>1 \mathrm{kpc}$. The central velocity dispersion during the merger roughly doubles and the old stellar disk is heated. The rotational support of the remnant disks remaining from gas-rich minor mergers is considerably larger than that measured for comparable gas-poor minor mergers by Bournaud et al. (2005), who calculate $V_{\text {rot }} / \sigma \approx 1.6$ for $8: 1$ mass-ratio gaspoor mergers.

The simulations confirm that merger-driven disk formation from gas-rich encounters at high redshift may be possible and highlight the role of energetic feedback on the rotational support of extremely gas-rich merger remnants. Finally, to confirm the merger-driven scenario for disk galaxy formation as a viable supplement to the dissipational collapse model, cosmological simulations of galaxy formation including pressurized multiphase ISM physics should be performed at sufficient resolution to determine the importance of early, gasdominated mergers to the cosmological frequency of disk galaxies. While cosmological simulations of galaxy formation with multiphase ISM physics appear promising (Robertson et al. 2004), simulations with sufficient resolution to track early interactions between gas-rich systems may provide a more comprehensive picture of cosmological disk galaxy formation.

This work was supported in part by NSF grants ACI 96-19019, AST 00-71019, AST 02-06299, and AST 0307690, and NASA ATP grants NAG5-12140, NAG513292, and NAG5-13381. The simulations were performed at the Center for Parallel Astrophysical Computing at Harvard-Smithsonian Center for Astrophysics.

\section{REFERENCES}

Abadi, M. G., Navarro, J. F., Steinmetz, M., \& Eke, V. R. 2003a ApJ, 591, 499

-. 2003b, ApJ, 597, 21

Barnes, J., \& Efstathiou, G. 1987, ApJ, 319, 575

Barnes, J. E. 1992, ApJ, 393, 484

-. 2002, MNRAS, 333, 481

Barnes, J. E., \& Hernquist, L. 1996, ApJ, 471, 115

Barnes, J. E., \& Hernquist, L. E. 1991, ApJ, 370, L65

Benson, A. J., Lacey, C. G., Frenk, C. S., Baugh, C. M., \& Cole, S. 2004, MNRAS, 351, 1215

Black, J. H. 1981, MNRAS, 197, 553

Blumenthal, G. R., Faber, S. M., Flores, R., \& Primack, J. R. 1986, ApJ, 301, 27

Blumenthal, G. R., Faber, S. M., Primack, J. R., \& Rees, M. J. 1984, Nature, 311, 517

Bournaud, F., Combes, F., \& Jog, C. J. 2004, A\&A, 418, L27

Bournaud, F., Jog, C. J., \& Combes, F. 2005, A\&A, 437, 69

Brook, C., Veilleux, V., Kawata, D., Martel, H., \& Gibson, B. 2005, in Island Universes: Structure and Evolution of Disk Galaxies, astro-ph/0511002

Brook, C. B., Kawata, D., Gibson, B. K., \& Freeman, K. C. 2004, ApJ, 612, 894

-. 2006, ApJ, 639

Bullock, J. S., Dekel, A., Kolatt, T. S., Kravtsov, A. V., Klypin, A. A., Porciani, C., \& Primack, J. R. 2001, ApJ, 555, 240

Cox, T. J., , Dutta, S. N., Di Matteo, T., Hernquist, L., Hopkins, P. F., Robertson, B., \& Springel, V. 2006, ApJ, submitted

de Vaucouleurs, G. 1948, Annales d'Astrophysique, 11, 247

Di Matteo, T., Springel, V., \& Hernquist, L. 2005, Nature, 433, 604

Djorgovski, S., \& Davis, M. 1987, ApJ, 313, 59
D’Onghia, E., \& Burkert, A. 2004, ApJ, 612, L13

Dressler, A., Lynden-Bell, D., Burstein, D., Davies, R. L., Faber,

S. M., Terlevich, R., \& Wegner, G. 1987, ApJ, 313, 42

Fall, S. M., \& Efstathiou, G. 1980, MNRAS, 193, 189

Font, A. S., Navarro, J. F., Stadel, J., \& Quinn, T. 2001, ApJ, 563, L1

Gingold, R. A., \& Monaghan, J. J. 1977, MNRAS, 181, 375

Governato, F., et al. 2004, ApJ, 607, 688

Hernquist, L. 1989, Nature, 340, 687

-. 1990, ApJ, 356, 359

Hernquist, L., \& Barnes, J. E. 1991, Nature, 354, 210

Hernquist, L., \& Mihos, J. C. 1995, ApJ, 448, 41

Hernquist, L., Spergel, D. N., \& Heyl, J. S. 1993, ApJ, 416, 415

Hopkins, P. F., Hernquist, L., Cox, T. J., Di Matteo, T., Martini, P., Robertson, B., \& Springel, V. 2005a, ApJ, 630, 705

Hopkins, P. F., Hernquist, L., Cox, T. J., Di Matteo, T., Robertson, B., \& Springel, V. 2005b, ApjS, in press

Hopkins, P. F., Hernquist, L., Cox, T. J., Robertson, B., \& Springel, V. 2005c, ApJ, in press

Hopkins, P. F., Hernquist, L., Martini, P., Cox, T. J., Robertson,

B., Di Matteo, T., \& Springel, V. 2005d, ApJ, 625, L71

Hultman, J., \& Pharasyn, A. 1999, A\&A, 347, 769

Katz, N., \& Gunn, J. E. 1991, ApJ, 377, 365

Katz, N., Hernquist, L., \& Weinberg, D. H. 1992, ApJ, 399, L109

Kennicutt, R. C. 1998, ApJ, 498, 541

Lacey, C., \& Cole, S. 1993, MNRAS, 262, 627

Lucy, L. B. 1977, AJ, 82, 1013

Maller, A. H., \& Dekel, A. 2002, MNRAS, 335, 487

McKee, C. F., \& Ostriker, J. P. 1977, ApJ, 218, 148

Mihos, J. C., \& Hernquist, L. 1994a, ApJ, 427, 112

-. 1994b, ApJ, 425, L13 
-. 1994c, ApJ, 431, L9

-. 1996, ApJ, 464, 641

Mo, H. J., Mao, S., \& White, S. D. M. 1998, MNRAS, 295, 319

Navarro, J. F., Frenk, C. S., \& White, S. D. M. 1997, ApJ, 490, 493

Navarro, J. F., \& Steinmetz, M. 2000, ApJ, 538

Navarro, J. F. \& White, S. D. M. 1994, MNRAS, 267, 401

Okamoto, T., Eke, V. R., Frenk, C. S., \& Jenkins, A. 2005, MNRAS, 363, 1299

Peebles, P. J. E. 1969, ApJ, 155, 393

Quinn, P. J., Hernquist, L., \& Fullagar, D. P. 1993, ApJ, 403, 74

Robertson, B., Cox, T. J., Hernquist, L., Franx, M., Hopkins, P. F., Martini, P., \& Springel, V. 2006, ApJ, 641

Robertson, B., Yoshida, N., Springel, V., \& Hernquist, L. 2004, ApJ, 606, 32

Sommer-Larsen, J., Götz, M., \& Portinari, L. 2003, ApJ, 596, 47

Sommer-Larsen, J., Gelato, S., \& Vedel, H. 1999, ApJ, 519, 501

Springel, V., Di Matteo, T., \& Hernquist, L. 2005a, ApJ, 620, L79 —. 2005b, MNRAS, 361, 776

Springel, V., \& Hernquist, L. 2003, MNRAS, 339, 289

-. 2005, ApJ, 622, 9

Steinmetz, M., \& Muller, E. 1995, MNRAS, 276, 549

Thacker, R. J., \& Couchman, H. M. P. 2000, ApJ, 545, 728

-. 2001, ApJ, 555, L17
Thakar, A. R., \& Ryden, B. S. 1996, ApJ, 461, 55

-. 1998, ApJ, 506, 93

Toomre, A. 1964, ApJ, 139, 1217

Toomre, A. 1977, in Evolution of Galaxies and Stellar Populations, $401-+$

Toth, G., \& Ostriker, J. P. 1992, ApJ, 389, 5

van den Bosch, F. C. 1998, ApJ, 507, 601

-. 2001, MNRAS, 327, 1334

van den Bosch, F. C., Abel, T., Croft, R. A. C., Hernquist, L., \& White, S. D. M. 2002, ApJ, 576, 21

van den Bosch, F. C., Burkert, A., \& Swaters, R. A. 2001, MNRAS, 326,1205

Velazquez, H., \& White, S. D. M. 1999, MNRAS, 304, 254

Vitvitska, M., Klypin, A. A., Kravtsov, A. V., Wechsler, R. H., Primack, J. R., \& Bullock, J. S. 2002, ApJ, 581, 799

Walker, I. R., Mihos, J. C., \& Hernquist, L. 1996, ApJ, 460, 121

Weil, M. L., Eke, V. R., \& Efstathiou, G. 1998, MNRAS, 300, 773

White, S. D. M., \& Rees, M. J. 1978, MNRAS, 183, 341

Yepes, G., Kates, R., Khokhlov, A., \& Klypin, A. 1997, MNRAS, 284, 235

Yoachim, P., \& Dalcanton, J. J. 2005, ApJ, 624, 701

—. 2006, AJ, 131, 226 